\title{
Progresses in the studies of adiabatic splitting of charged particle beams by crossing nonlinear resonances
}

\author{
A. Franchi, S. Gilardoni, and M. Giovannozzi \\ CERN, Geneva, Switzerland \\ (Received 17 July 2008; published 20 January 2009)
}

\begin{abstract}
The multiturn extraction from a circular particle accelerator is performed by trapping the beam inside stable islands of the horizontal phase space. In general, by crossing a resonance of order $n, n+1$ beamlets are created whenever the resonance is stable, whereas if the resonance is unstable the beam is split in $n$ parts. Islands are generated by nonlinear magnetic fields, whereas the trapping is realized by means of a given tune variation so to cross adiabatically a resonance. Experiments at the CERN Proton Synchrotron carried out in 2007 gave the evidence of protons trapped in stable islands while crossing the one-third and one-fifth resonances. Dedicated experiments were also carried out to study the trapping process and its reversibility properties. The results of these measurement campaigns are presented and discussed in this paper.
\end{abstract}

DOI: 10.1103/PhysRevSTAB.12.014001

PACS numbers: 29.27.Ac, 05.45.-a, 29.27.Bd

\section{INTRODUCTION}

In 2002 a new scheme was proposed to eject the beam from a circular particle accelerator over a few turns by means of nonlinear magnets, such as sextupoles and octupoles, rather than slicing it onto an electrostatic septum $[1,2]$. This scheme, tailored for a five-turn extraction and named multiturn extraction (MTE), is due to be commissioned at the CERN Proton Synchrotron (PS) this year [3]. The beam is split and trapped inside stable islands of the horizontal phase space, which are generated by sextupole and octupole magnets and separated by sweeping the horizontal tune through the one-fourth resonance, $Q_{x}=6.25$. This approach has several advantages compared with the beam shaving and separation through the electrostatic septum [4]. First, there is no interaction between the beam and the septum blade, whose losses limit highintensity operations. Second, at extraction the beamlets trapped in the islands have the same intensity, emittance and optical parameters, easing the matching with the downstream transfer line and accelerator [5]. This would not be possible by using an electrostatic septum. Third, several parameters, such as the nonlinear magnet strengths, the resonance crossing speed, and the final horizontal tune, are available to adjust and optimize simultaneously the beamlet parameters and separation. With the electrostatic septum, only the bump amplitude may be used to equalize either the intensities or the emittances of the five slices. It is worth noting the same scheme can be time reversed and used for a resonant multiturn injection. In Ref. [6] it has been proposed to inject beamlets over several turns directly into the stable islands. By crossing the resonance from the direction opposite to the one used for the MTE, the islands are moved towards the center. As shown in Ref. [6], and further discussed at the end of this paper, if no beamlet is injected directly at the center, the result of such a manipu- lation is a hollow beam, whose reduced peak intensity might be of great help for the injection of high-intensity beams.

The number of beamlets generated depends on the crossed resonance. In the case of the beam transfer between the CERN PS and the Super Proton Synchrotron (SPS) at $14 \mathrm{GeV} / c$, the most suitable resonance is the onefourth, $Q_{x}=6.25$ [2]. This choice is dictated by the need to minimize transient effects in the SPS, that should be filled as uniformly as possible. For decades two PS cycles are used to fill the SPS ring, whose circumference is 11 times the PS one. At the end of each PS cycle the beam is ejected in five turns. The SPS is then filled in 10/11 of its length, as one empty slot is left to avoid interference between the circulating beam and the transient times of the SPS kickers. This is the underlying idea of the present continuous transfer (CT) extraction [4], where the beam is shaved in five slices in five consecutive turns by means of an electrostatic septum. The optimal two-cycle structure was then kept also for the MTE.

Starting from 2002 extensive measurement campaigns have been carried out in order to assess the feasibility of a loss-free beam splitting by means of nonlinear magnets. Their success $[7,8]$ and the need of reducing losses during the production of high-intensity proton beams for the CERN Neutrino to Gran Sasso (CNGS) [9] experiment, led to the approval of the MTE project.

So far the splitting was experimentally proved in the CERN PS by crossing the stable one-fourth resonance, $Q_{x}=6.25$. However, both the theoretical model and numerical simulations indicate that other resonances, regardless their stability, may be crossed to generate different numbers of beamlets [2]. In general, by crossing adiabatically a resonance of the order $n, n+1$ beamlets are created whenever the resonance is stable, whereas if the resonance is unstable the beam is split in $n$ parts only. The resonance 
stability indeed affects the beam center only: an unstable resonance renders the origin an unstable fixed point, hence moving particles towards other stable fixed points (the islands) and depleting the beam core. Of course, dealing with single-particle instability makes the adiabatic capture more delicate, as part of the beam may be spilled out the beam core, and hence lost, before being properly captured in the stable islands.

The delicate point of the one-fourth resonance crossing concerns the equalization of the beam parameters between the four beamlets trapped in the islands and the one remaining in the central area. The latter is unavoidably ejected with root-mean-square (RMS) optical parameters slightly different from the ones of the islands. As far as the intensity is concerned, the best beam sharing so far achieved is of $\sim 19 \%$ in the four islands and $\sim 24 \%$ in the central core. A maximum difference of about $5 \%$ in the sharing between islands and core is required by the SPS in order to avoid unwanted transient effects and to guarantee a regular spill structure to the experiments. Since the equal sharing among core and islands is not granted by the dynamics of the islands formation, a time-consuming fine-tuning of all machine parameters is required at any change of the beam settings.

Another delicate point is the need of an additional kick to extract the beam core that might induce a slightly different trajectory in the transfer line, compared to the ones of the islands, not to mention the impact on the hardware requirements.

Dedicated experiments at the CERN PS were carried out in 2007 at $14 \mathrm{GeV} / c$ to exploit the possibility of generating islands by crossing other resonances: the unstable onethird, generating three beamlets only, and the stable onefifth, thus creating six beamlets, five in the islands plus the beam core. In the former case, three PS cycles might be used to fill the $9 / 11$ of the SPS. In the case of the one-fifth resonance, $N$ repeated crossings during the flattop of the same cycle can be envisaged, in order to extract $N$ batches of five identical beamlets each, see Ref. [2]. The beam remaining in the central area after $N$ crossing of the onefifth resonance might be eventually ejected towards a beam dump. In both cases, the beamlets ejected would have exactly the same intensities and RMS optical parameters.

Other aspects that have been experimentally investigated concern the adiabaticity (i.e. the preservation of the integrals of motion) and the reversibility (i.e. the capability of retrieving the initial conditions after reversing the process). Both are key ingredients to guarantee a robust and efficient particle trapping around the fixed points created by the nonlinear elements. Indeed, while the horizontal tune moves away from the resonance, the distance between the fixed points and the central region of the phase space increases. A too fast crossing would prevent particles from following the fixed points, thus reducing the amount of beam trapped. On the other hand, operational constraints, such as the overall duration of the accelerator magnetic cycle, do not allow an arbitrarily long flattop, which need to be shared between the splitting and other beam manipulations. As far as the CERN PS is concerned, the time available for the beam splitting will be of $50-90 \mathrm{~ms}$, corresponding to about $24-34 \times 10^{3}$ turns at $14 \mathrm{GeV} / c$. Hence, it is of interest to define the minimum time required to cross the resonance so to leave the final beam parameters unaffected. It is worthwhile mentioning that a simplified 2D Hénon-like model [10] of Ref. [2] predicts a complete reversibility (and hence adiabaticity) of the process for a sufficiently large crossing time $T^{*} \sim 200 \mathrm{~ms}\left(\sim 10^{5}\right.$ turns). This however does not include neither the small, albeit non-negligible, nonlinear coupling between the two transverse planes introduced by sextupoles and octupoles, nor the coupling with the synchrotron motion, the horizontal tune being modulated by the synchrotron oscillations via the natural chromaticity.

This paper aims at presenting (i) the first experimental evidences of beam splitting by crossing the unstable onethird and the stable one-fifth resonances in the CERN PS, and (ii) the studies on the adiabaticity and reversibility of the capture process. Results on the splitting are compared with numerical predictions making use of the most realistic PS lattice model.

For the sake of completeness, it is worth mentioning that experimental studies on the crossing of the one-third resonance in an electron machine were recently reported in Ref. [11].

The plan is the following: in Sec. II the PS numerical model is presented. In Sec. III the experimental setup and results of beam trapping obtained in the 2007 run by crossing the above resonances are summarized and discussed. The analysis of the experimental observations on the adiabaticity and reversibility is eventually presented in Sec. IV.

\section{THE PS NUMERICAL MODEL}

The PS lattice consists of ten super periods each made of ten combined function dipole magnets $4.4 \mathrm{~m}$ long, interlaced with eight $1.6 \mathrm{~m}$ and two $3.0 \mathrm{~m}$ straight sections (SS) [12]. Every magnet is composed of two half units with gradients of opposite sign, separated by a central junction. The latest magnetic measurements using Hall probes [13] showed that stray fields at the magnet ends introduce an additional quadrupolar component, while in the gap between the two half units a sextupolar component was observed. The fine adjustment of tunes and chromaticities is performed by means of extra coils mounted on the pole faces of the main dipoles (pole-face windings). Until 2007 this system was controlled by three independent currents, sufficient to set both tunes and the horizontal chromaticity. Towards the end of 2007 an upgraded version was commissioned, able to control independently five physical parameters (such as tunes, chromaticities plus one addi- 


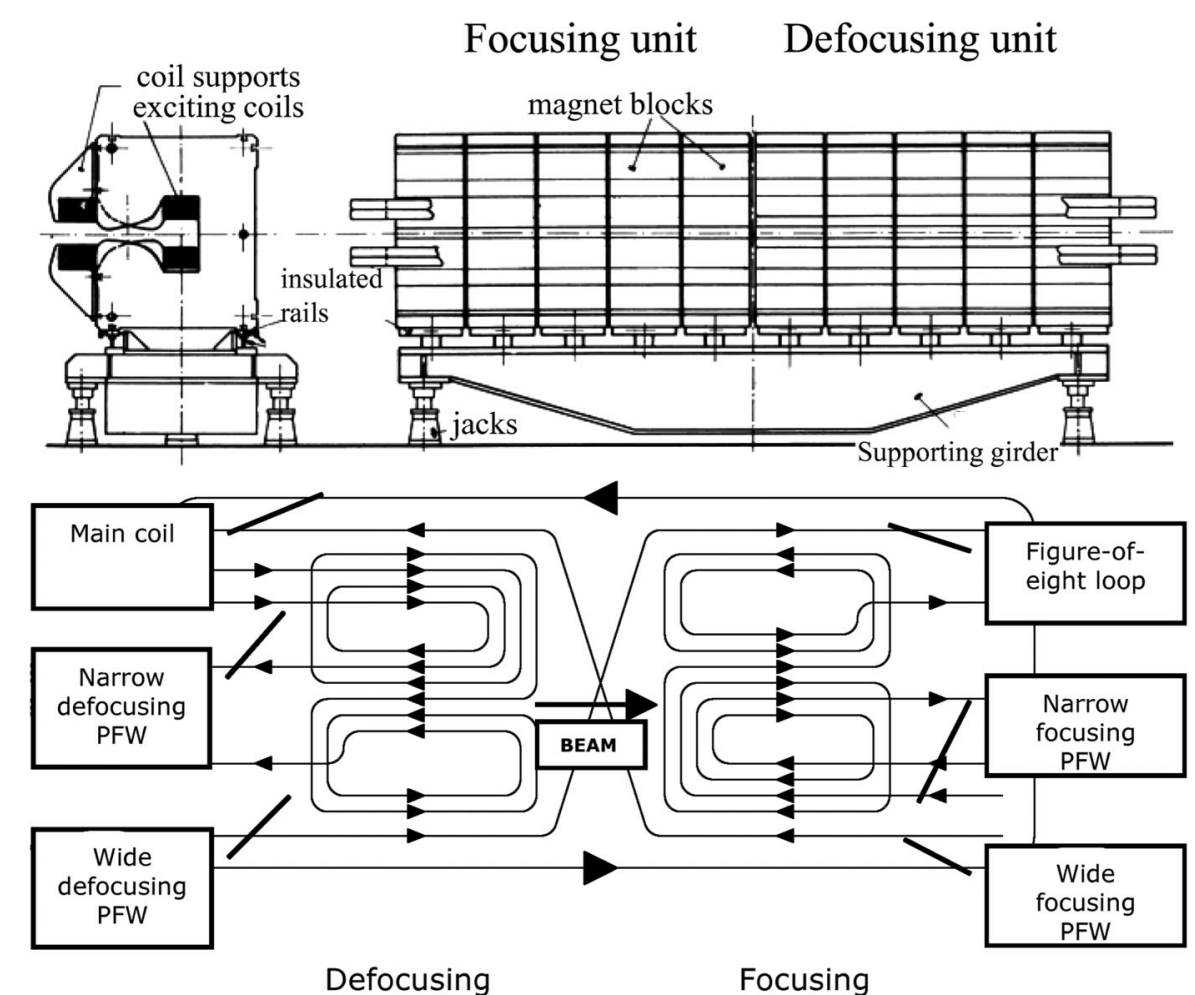

FIG. 1. Sketch of PS main magnet (upper) and of the five circuits used to control the tunes and the chromaticities (lower), from Ref. [22].

tional free parameter) by means of five new separate circuits [14]. A sketch of the PS main magnet is shown in the upper part of Fig. 1, while the schematic view of the five circuits to control tunes and chromaticities are shown in the lower part.

The PS numerical model is then constructed as follows. The dipole and quadrupole fields generated by the main combined function magnets is fixed by the beam momentum. The additional quadrupolar components induced by stray fields and pole-face windings are modeled by quadrupole thin lenses placed at the half-unit ends. Sextupolar and higher-order fields generated by the pole-face windings and by the intermagnet gap are eventually represented

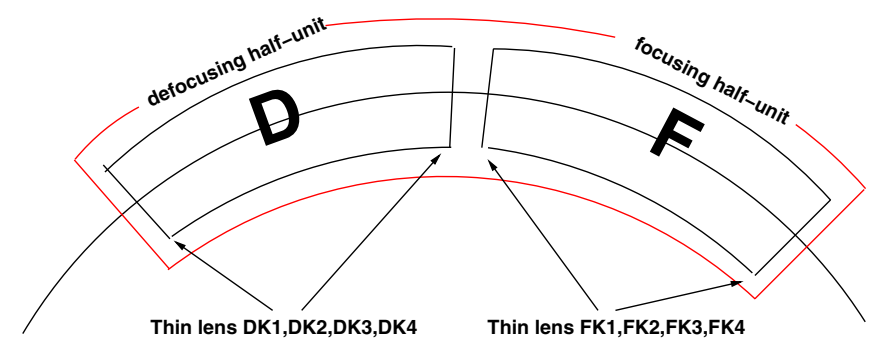

FIG. 2. (Color) Schematic picture of the PS magnet model as of 2007. K1, K2, K3, and K4 denote the quadrupole, sextupole, octupole, and decapole thin lenses, respectively. The prefixes D and $\mathrm{F}$ distinguish between the focusing and defocusing magnet families. by nonlinear thin lenses, placed at the half-unit ends. The thin lenses so introduced are eventually grouped in two families, for the focusing and the defocusing half units, respectively, as sketched in Fig. 2. Having the pole-face windings an effects on global parameters (tunes and chromaticities), it was decided to represent the five circuits by two effective families of thin multipoles only.

The effective lattice model is then computed by measuring nonlinear chromaticity in both planes at the desired working point and energy. This beam-based technique has been implemented in the PS since 2002 [15]. The transverse tunes are measured as functions of the momentum offset generated by a proper radio-frequency perturbation. A polynomial fit of the measured curves is performed to extract numerical information on the different magnetic multipole orders. Then, the integrated strengths of the above thin-lenses elements, $K_{n}=1 /\left(B_{0} \rho_{0}\right) \partial^{n} B_{y} / \partial x^{n}$, where $B_{0} \rho_{0}$ is the magnetic rigidity, are computed to match the curves. This procedure is applied order by order, i.e. the quadrupole components are used to reproduce the constant term in the polynomial, the sextupole components the linear term and so on, up to the decapolar components. The two families (defocusing and focusing) are used to match each quantity in both planes.

An example of chromaticity measurement and polynomial fit is shown in Fig. 3. The two families of thin-lenses elements have been matched with the polymorphic tracking code (PTC) module of MAD-X [16,17] in order to 

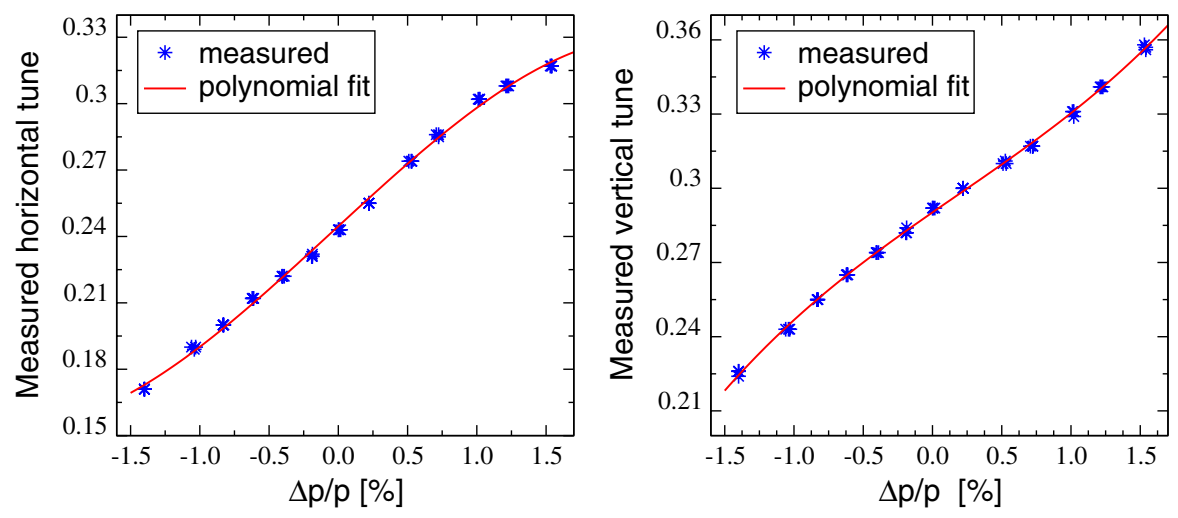

FIG. 3. (Color) Example of measured chromaticity and polynomial fit in the PS at $14 \mathrm{GeV} / c$.

reproduce the measured curve. Unless specified, all measurement results presented here refer to data taken in 2007 before the pole-face windings upgrade. PTC was also used to perform multiparticle simulations of the beam trapping.

\section{EXPERIMENTAL RESULTS ON BEAM TRAPPING}

The magnetic elements and the beam instrumentation used in the experimental campaign are shown in Fig. 4. The tune at $14 \mathrm{GeV} / c$ is changed so to cross a resonance by means of two families of focusing and defocusing quadrupoles, normally used to tune the machine at injection energy. Two sextupoles and one octupole located in the straight section (SS) 21 are used to generate the stable islands. Flying wire scanners, whose characteristics and features can be found in Refs. [7,18], are then used to measure the horizontal beam distribution and to monitor its evolution during the resonance crossing. As depicted in Fig. 4, only two horizontal flying wire scanners are avail-

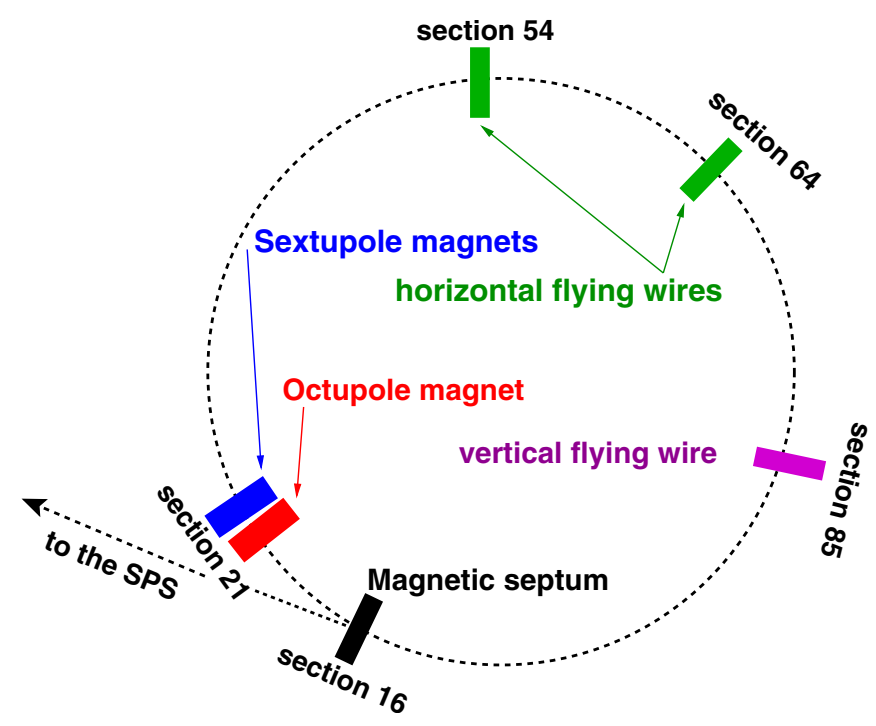

FIG. 4. (Color) Schematic layout of the PS ring with the elements used during the experiments. able (in SS54 and SS64) to measure the horizontal beam profile. This prevents the direct measurement of the horizontal phase space through a tomographic reconstruction, the latter requiring at least three profiles. Unless specified, measurements of the horizontal beam profile were done by means of the device in SS54.

The determination of islands' position, beamlets' size, and fraction of trapped particles is inferred from the recorded beam profile. This is performed by fitting $n$ or $n+$ 1 Gaussian functions, according to the resonance order $n$ and its stability type. The functions used to fit the islands are constrained to have the same intensity. The reason for such a constrain is twofold: First, the resonance symmetry guarantees that all islands have the same probability of trapping particles. Second, each measured island profile is actually an average over all islands profiles, the wire time of flight being much longer than the revolution period $(2.1 \mu \mathrm{s})$ and the islands swapping position turn by turn. Further details on the fitting procedure can be found in Ref. [7].

The PS is also equipped with several beam loss monitors (BLMs) whose signals can be acquired every $1 \mathrm{~ms}$, even though data taken during the measurements presented here were sampled every $10 \mathrm{~ms}$. The system is composed by 100 aluminum cathode electron multiplier (ACEM) detectors mounted on top of the main magnets. The signals are sampled and integrated by an 8 bit card, hence making the BLM saturate whenever the monitor counts are greater than 256. A proper calibration of the counts against beam loss is not available for a quantitative assessment of localized losses. More details on the BLM system can be found in Refs. $[19,20]$.

The beam current transformer used to measure the beam intensity during the experiments has a resolution of $2.5 \times$ $10^{10}$ protons, corresponding to less than about $1 \%$ for the beam intensities of the measurements presented here.

\section{A. Beam splitting by crossing the one-third resonance}

Three stable islands were created for the first time on August 10, 2007, using a single bunch of $\sim 3 \times 10^{12}$ pro- 

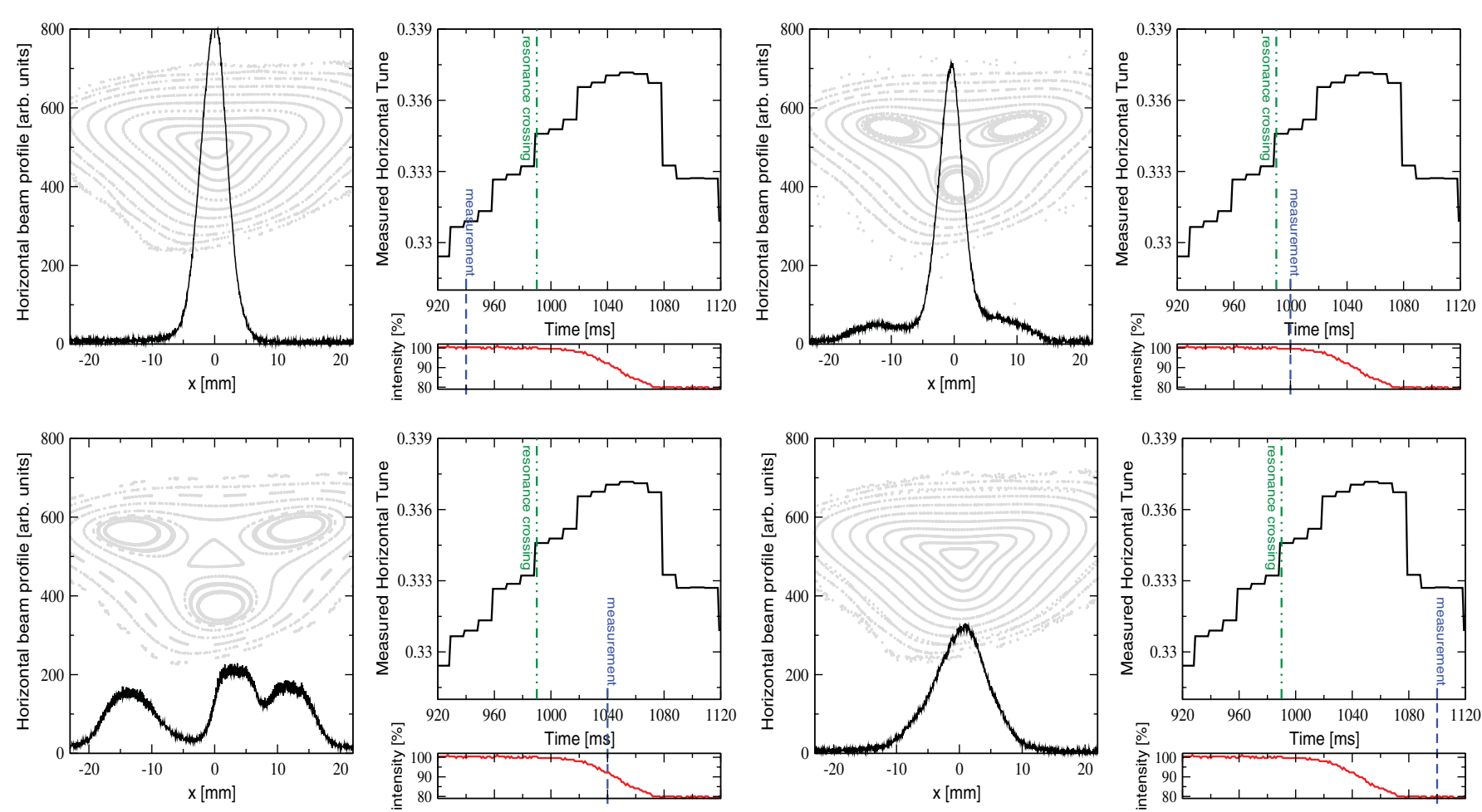

FIG. 5. (Color) Evolution of the horizontal beam profile during the crossing of the one-third resonance. The profile is shown before the resonance is crossed (upper left), right after the crossing (upper right), at the end of the crossing (lower left), and when the horizontal tune is brought back on the resonance (lower right). The corresponding horizontal phase space portrait obtained from the MAD-X PS model is in the background (gray) of the measured profile.

tons, whose horizontal RMS normalized emittance at $1 \sigma$ before the resonance crossing was of $5.7 \mu \mathrm{m}$. The horizontal tune was initially set to 6.330 , then increased linearly until the value of 6.337 in about $100 \mathrm{~ms}\left(\sim 4.8 \times 10^{4}\right.$ turns), and eventually brought back to the initial value, as depicted in the plots of Fig. 5. The vertical tune was set to 6.205 throughout the double resonance crossing.

Both the octupole and the sextupoles were powered with a constant current of $-421 \mathrm{~A}\left(K_{3}=-155 \mathrm{~m}^{-3}\right)$ and $330 \mathrm{~A}\left(K_{2}=0.9 \mathrm{~m}^{-2}\right)$, respectively. The main results are plotted in Fig. 5, where the horizontal beam profile is shown before the resonance is crossed (upper left), right after the crossing (upper right), the end of the crossing (lower left), and when the horizontal tune is brought back on the resonance (lower right). Next to each profile the corresponding position on the tune ramp is indicated. In the background of the measured profile, in gray, the horizontal phase space portrait computed by the MAD-X program for the corresponding working point is shown. For completeness, below the window with the curve of the horizontal tune the temporal evolution of the beam intensity is reported. While the resonance is crossed without measurable losses, at the end of the capture a loss of about $10 \%$ occurs around $1020 \mathrm{~ms}$ (even though the basic PS magnetic cycle lasts $1200 \mathrm{~ms}$, a special $2400 \mathrm{~ms}$ long cycle was used for these measurements). The steep descent of the tune after $1050 \mathrm{~ms}$ might be the reason of the further beam loss, that at the end of the process is of about 20\%. From the measured profiles it is not possible to conclude whether losses occur because particles trapped in the islands drift away as for the normal slow extraction, or whether they are due to untrapped (or detrapped) particles in the chaotic region between the core and the islands. Nevertheless, BLM data clearly show that losses are not uniformly distributed along the machine, but rather localized in three regions with reduced horizontal aperture. In Fig. 6 the loss pattern along the PS ring is shown for the entire duration of the resonance crossing. The most severe losses (in gray in the picture, at about $150 \mathrm{~m}, 255 \mathrm{~m}$ and $400 \mathrm{~m}$, respectively) occur in three regions corresponding to horizontal aperture restrictions introduced by an extraction septum, an injection septum, and the flying wire scanner tank, respectively. Protons that are scattered by those elements might be the source of the losses recorded in their proximity downstream. In the rest of the machine only small or no losses have been observed. The nonzero BLM signals prior the resonance crossing, before $940 \mathrm{~ms}$, account for injection losses, since the BLMs integrate the signal in time. The fact that other regions with reduced horizontal aperture do not show measurable losses might be due to an interplay between the islands displacement and the orbit distortion along the machine, that could be neither measured nor optimized during the experiment. In fact, the orbit measurement system cannot provide a reliable orbit measure- 

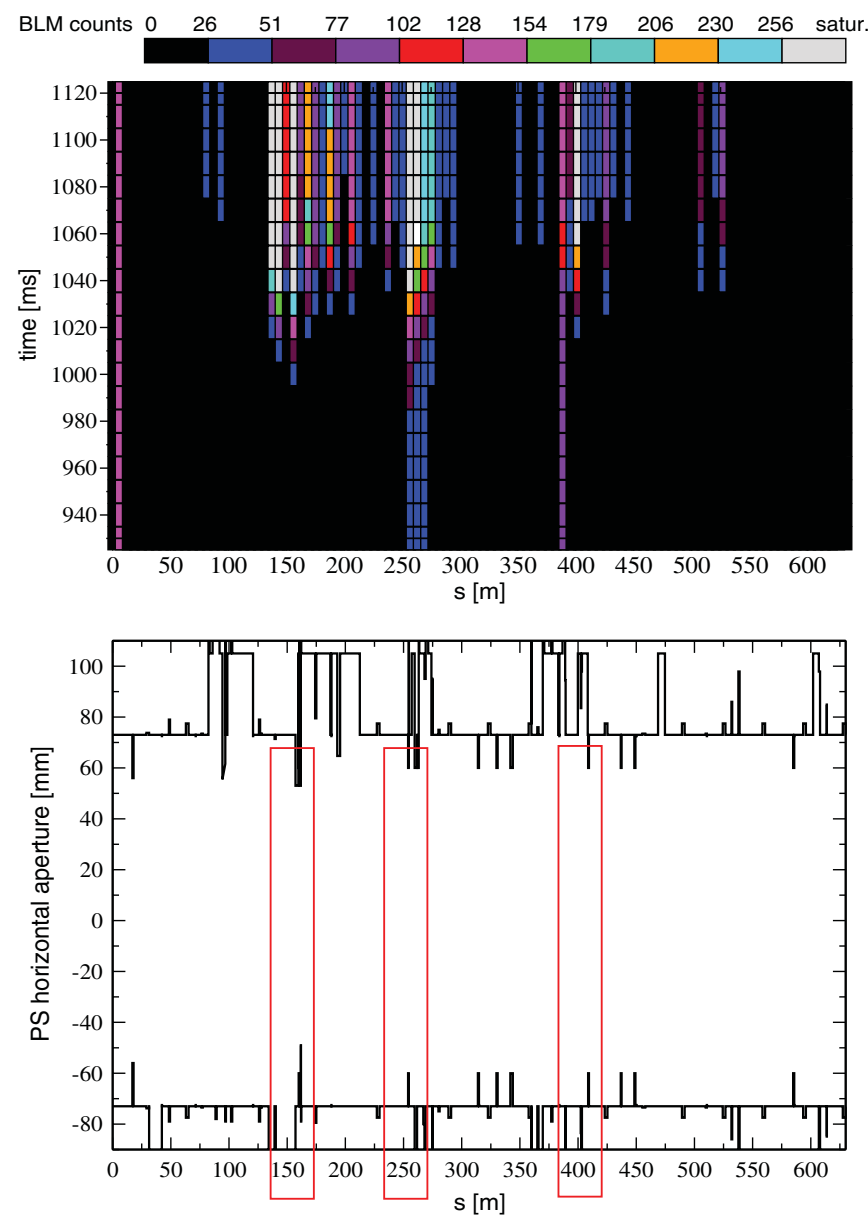

FIG. 6. (Color) Top: measurements of beam loss along the PS circumference $(\sim 628 \mathrm{~m})$ versus time, zoomed in the temporal window corresponding to the resonance crossing, which occurs at $990 \mathrm{~ms}$. The colors refer to the BLM reading that saturates after reaching 256. Bottom: PS horizontal aperture model. Important losses are localized in three regions with aperture reduction induced by septa and special vacuum chambers.

ment in the presence of islands: Their oscillations at large amplitudes and the almost empty beam core, indeed, make it impossible to obtain quantitative information on the beamlets' trajectories.

The above considerations indicate that the observed beam loss is not an intrinsic feature of the crossing of an unstable resonance. By correcting the closed orbit, optimizing the islands' position through a proper setting of the tune and of the nonlinear elements, and correcting chromaticity, a lossless capture is expected, in agreement with numerical simulations [2].

The beam profiles measured during the capture were fitted with a superposition of four Gaussian functions. An example is shown in Fig. 7. The beam profile measured at the end of the capture $(1070 \mathrm{~ms})$ is well fitted by three main Gaussian curves (green in the picture), each one containing a fraction of particle of about $31.8 \%$. The remaining $4.6 \%$ of the beam remains in the central area (red in the picture).

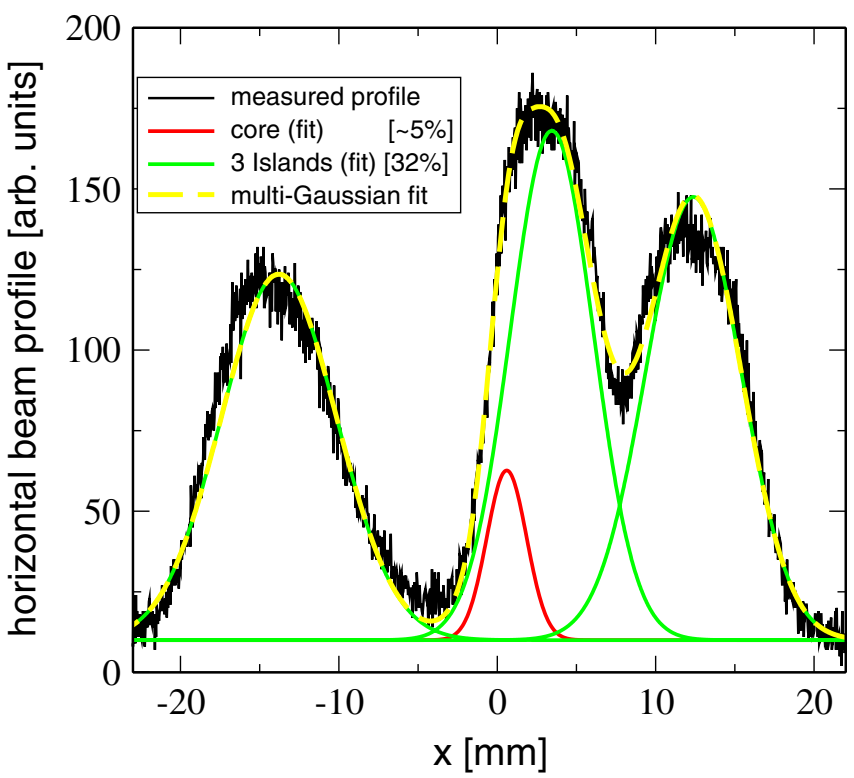

FIG. 7. (Color) Measured horizontal beam profile at the end of the capture with superimposed multi-Gaussian fit.

Both the 2D Hénon-like model of Ref. [2] and pure 4D multiparticle simulations predict a complete depletion of the beam core and well separated islands, see left plot of Fig. 8. This is not the case when chromatic effects $\left(Q_{x}^{\prime}=\right.$ 1.2) and tune modulation induced by the synchrotron motion (synchrotron tune $Q_{s}=1 \times 10^{-2}$ and momentum spread $\Delta p / p=1 \times 10^{-3}$ at $\left.2 \sigma\right)$ are included: Simulations in this case show that about $6 \%$ of the beam remains in the core, while about another $6 \%$ is confined in the region between islands and core, see right plot of Fig. 8. None of the above simulations showed however particles lost. A further check on the beam energy during the experiment revealed a momentum offset of $1.4 \times 10^{-3}$. By including the latter in simulations, a mere $1 \%$ of the beam is predicted to be lost. Unfortunately, it was not possible to assess whether the jumps in the measured linear tune ramp set to cross the resonance (see Fig. 5) were an artifact of the measurement system. Nonetheless the simulations were performed assuming an ideal linear ramp between 920 and $1070 \mathrm{~ms}$.

In Fig. 9 the temporal evolution of the main beamlets' parameters, inferred from the multi-Gaussian fit, is reported. The three islands are clearly generated between 980 and $990 \mathrm{~ms}$, i.e., right after the resonance crossing. From 980 to $1010 \mathrm{~ms}\left(Q_{x}=6.335\right)$ the islands increase their separation and population and no losses are detected. At the end of this interval, about $94 \%$ of the particles get trapped in the islands. Losses appear in the second part of the tune ramp. Starting from $1070 \mathrm{~ms}$ the horizontal tune is rapidly brought back to the initial value in $20 \mathrm{~ms}$, corresponding to about 9500 turns: The islands' move back towards the center to merge eventually with the beam core. The factor two between final RMS beam size, 

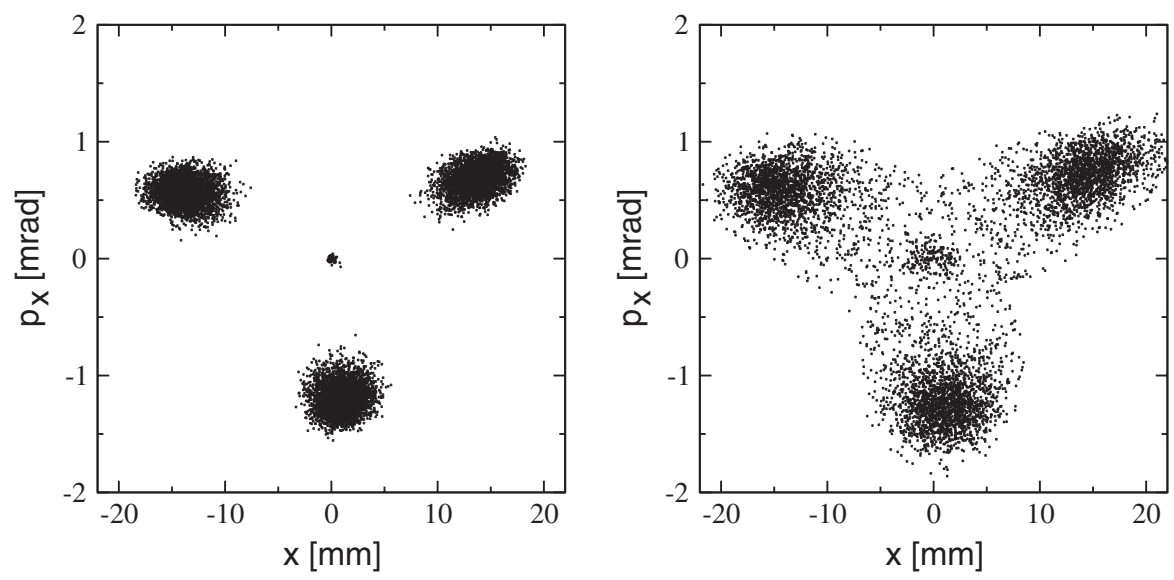

FIG. 8. Phase space at the end of the one-third resonance crossing from multiparticle simulations of the experiment. If pure 4D tracking is performed the three islands are well separated and the core is almost empty (left plot), whereas if the horizontal tune modulation driven by chromaticity is included, $6 \%$ of the beam remains in the core and about the same fraction remains in the region between the islands (right plot).

$\sigma_{x, f}=4.5 \mathrm{~mm}$, and the initial one, $\sigma_{x, i}=2.14 \mathrm{~mm}$, see Fig. 5) might be explained by the rapid inverse crossing and the particles confined in the region between islands and core during the first crossing.

\section{B. Beam splitting by crossing the one-fifth resonance}

A similar manipulation was performed on July 27th, when five stable islands were created for the first time. The beam parameters were the same of the one-third resonance crossing. The horizontal tune was initially set to 6.190 , then increased linearly until the value of 6.213 in about $300 \mathrm{~ms}\left(\sim 1.3 \times 10^{5}\right.$ turns $)$, as depicted in the upper

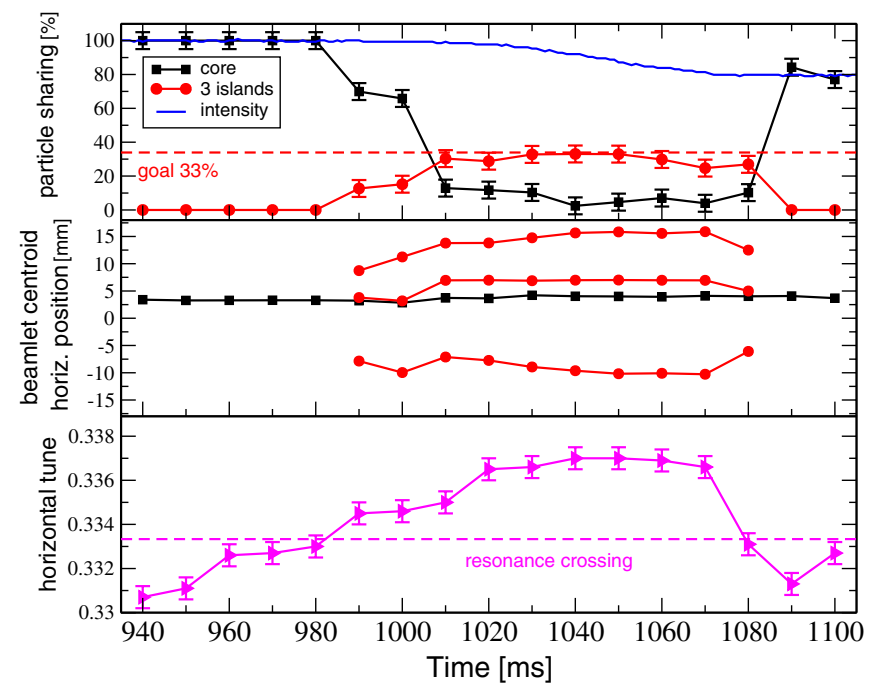

FIG. 9. (Color) Temporal evolution of the main beamlets' parameters: particle sharing among the core and the three islands (top) and the islands' horizontal centroid, i.e., fixed points (center). For completeness the horizontal tune variation is also displayed (bottom).
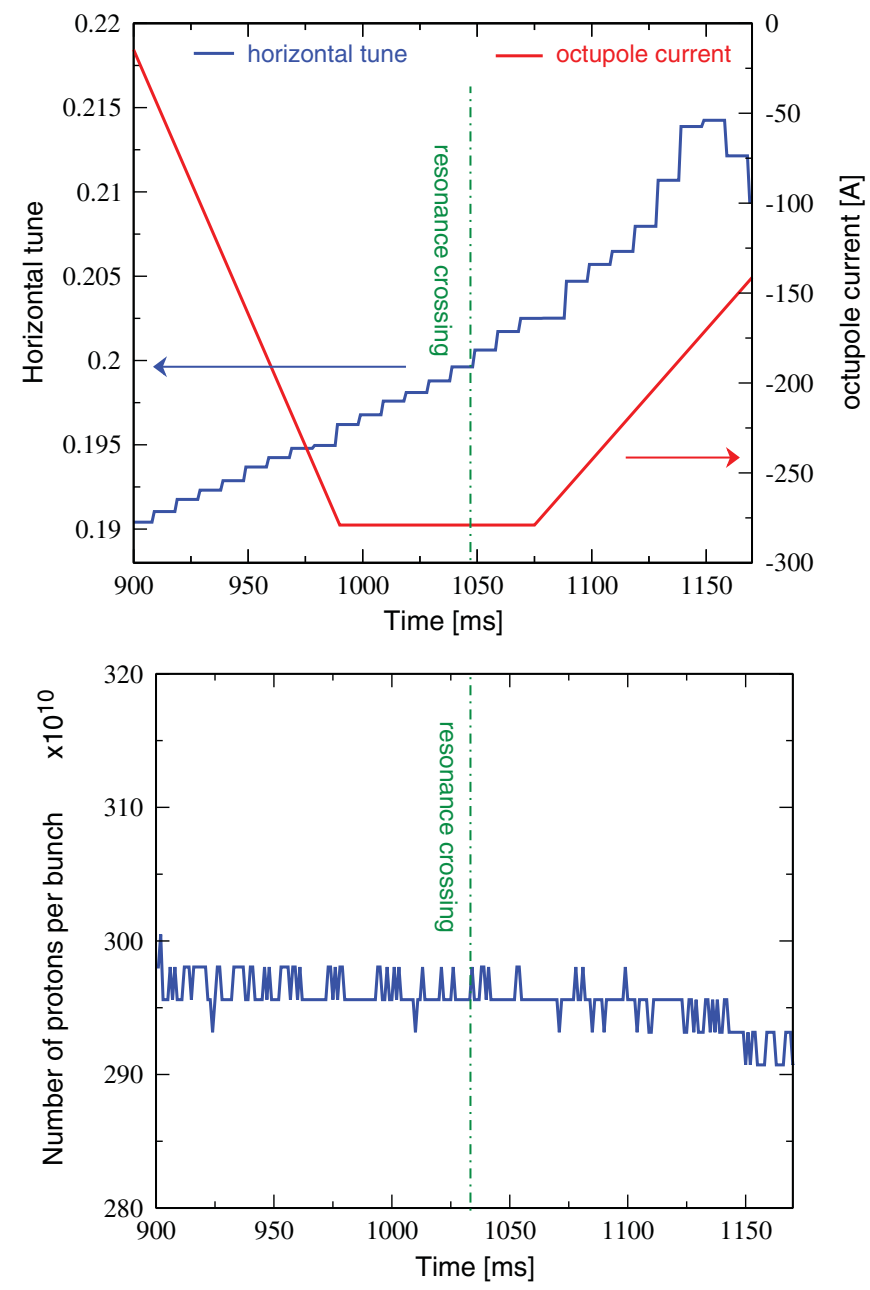

FIG. 10. (Color) Top: measured horizontal fractional tune and temporal evolution of the octupole current in the interval where the one-fifth resonance is crossed. Bottom: number of protons per pulse measured by the current transformer during the crossing of the one-fifth resonance. 
plot of Fig. 10. The vertical tune was kept constant to about 6.3 throughout the splitting process. Unlike the one-third resonance crossing experiment, the octupole was programmed to impart a large gradient $K_{3}=99 \mathrm{~m}^{-3}$ ( $-280 \mathrm{~A})$ during a fraction of the crossing $(85 \mathrm{~ms})$, to be then reduced at the end of process to $K_{3}=15 \mathrm{~m}^{-3}$ $(-40 \mathrm{~A})$. The sextupole current was fixed to $350 \mathrm{~A}\left(K_{2}=\right.$ $1.0 \mathrm{~m}^{-2}$ ) in order not to perturb the machine chromaticity.

Data from the current transformer clearly show that no losses occur throughout the resonance crossing, the beam intensity remaining constant at about $\sim 2.95 \times 10^{12}$ (see right plot of Fig. 10). In general the phase space topology, namely the islands' position, depends on both the value of the horizontal tune and the octupole strength. For a given magnet current, the farther is the tune from the resonant condition $\left(Q_{x}=6.20\right.$ in this case), the more distant are the islands. On the other hand, the same effect is obtained by fixing the working point at the end of crossing and reducing the octupole gradient $K_{3}$. Mathematical relations between tune, nonlinear magnets' strength, and island topology were already derived in Ref. [8] for the one-fourth resonance, showing the same characteristic. Another general feature of the splitting process is that, while the islands' position and size depend on the actual values of tune and octupole gradient, their intensity, namely the fraction of captured particles, depends mainly on how the octupole
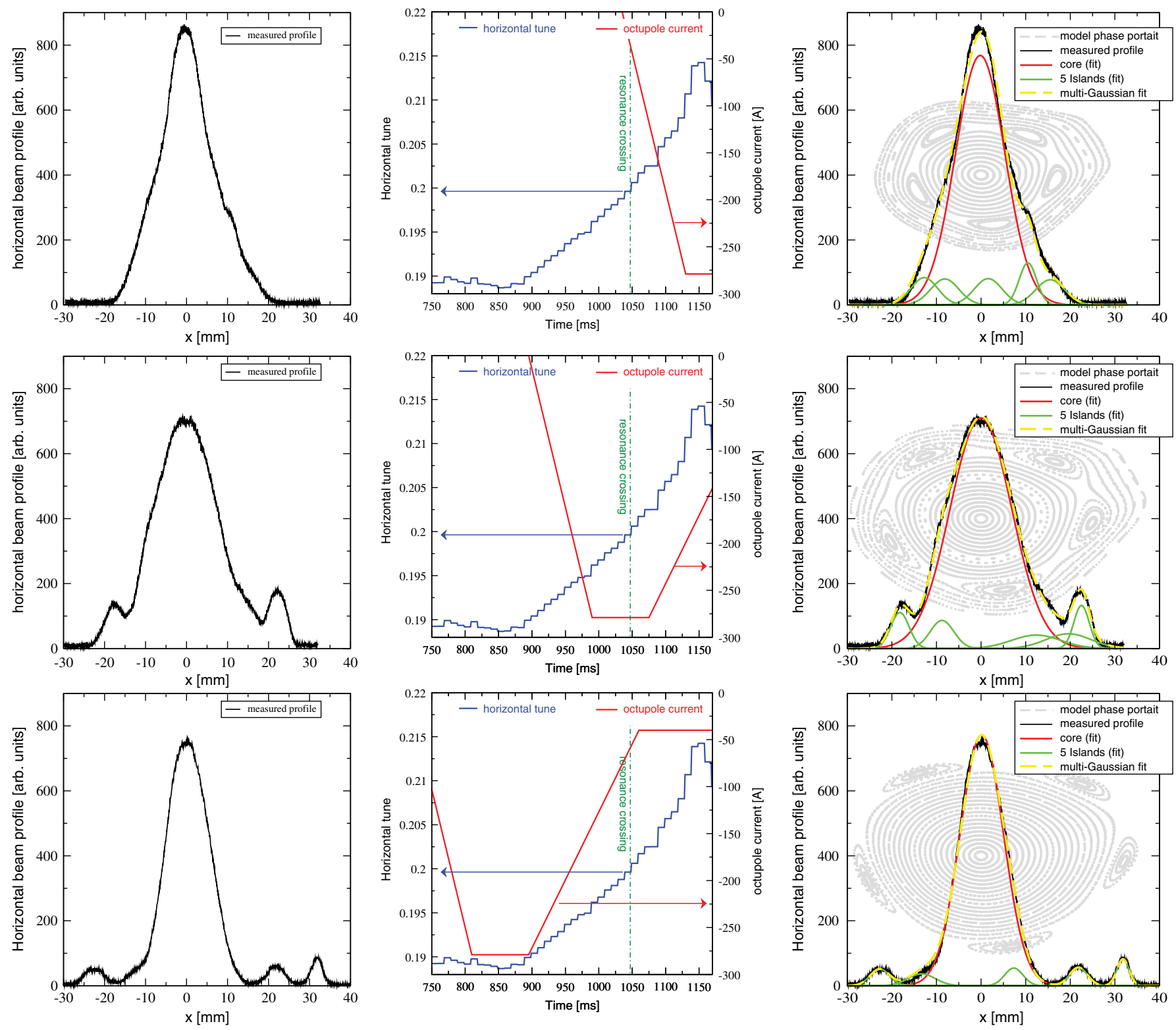

FIG. 11. (Color) Horizontal beam profiles (left) measured at the end of the one-fifth resonance crossing (1160 ms) for three different settings of the octupole currents (center). The multi-Gaussian fits and the corresponding phase space portraits computed from the numerical PS model are shown in the right plots. 
strength is programmed during the crossing. As already reported in Ref. [7], an octupole function as the one in the left plot of Fig. 10, provides a better particle sharing between islands and core and minimizes losses when compared with a crossing at constant octupole current.

A simple way to scan over the octupole strength without a repeated and time-consuming programming of the octupole current curve was found by delaying the octupole turn-on. Several measurements of the horizontal beam profile were taken at a fixed time $(1160 \mathrm{~ms})$ and tune $\left(Q_{x}=\right.$ 6.212), after retarding the octupole pulsing each time of $10 \mathrm{~ms}$. Three cases are shown in Fig. 11. In the first case (top row) the resonance is crossed (at $1050 \mathrm{~ms}$ ) with the octupole weakly powered, while the measurement is taken when its strength is maximum: five islands close to the beam center are then expected. In the second case (center row) the octupole is set to impart the maximum strength $(I=-280 \mathrm{~A})$ when the resonance is crossed. The profile in this case is measured when the octupole current is halved $(I=-140$ A) yielding more separated islands. In the third and last case (bottom row), the octupole curve is further shifted towards left and the resonance is crossed when the octupole current is of about $-50 \mathrm{~A}$. The lower current generates less populated, but more separated, islands.

These considerations are confirmed by displaying the phase space portraits computed by the MAD-X program on top of the measured profiles, and by performing the multiGaussian fit, as shown in the right pictures of Fig. 11. The agreement between the fitted Gaussian functions and the islands' position is excellent and unambiguous for the third case, when the islands are well separated. When instead the distance is reduced the fit may not be unique (first case), and the phase space portrait can be used to set some constraints, such as the island centroids.

The fraction of particles trapped in the islands is by far smaller than in the case of the one-third and one-fourth resonances (see Fig. 7 and Ref. [7], respectively). This feature was already predicted by multiparticle simulations of a simplified Hénon-like model in Ref. [2].

\section{STUDIES ON ADIABATICITY OF THE ISLAND CAPTURE}

\section{A. Measurement strategy}

Both the adiabaticity and the reversibility of the beam splitting were studied experimentally by setting up a long flattop during which the resonance is crossed twice, in opposite directions, as sketched in Fig. 12. The beam profile is then measured with a flying wire scanner at three different moments: before the first crossing (initial condition), after the first crossing (beamlets are generated and measured), and after the second crossing (beamlets are merged together and the final profile is compared to the first one). This procedure is then repeated for different resonance crossing times $T^{*}$, which are the same for both
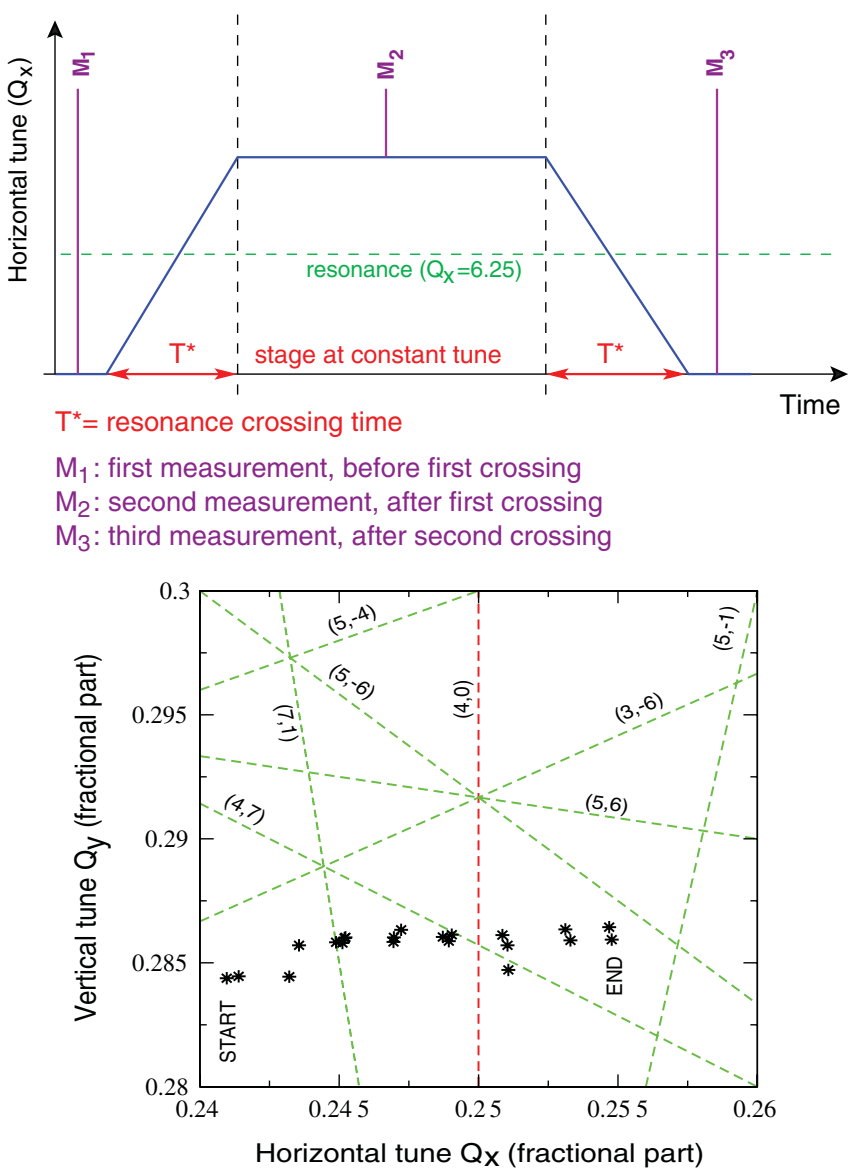

FIG. 12. (Color) Sketch of the measurement procedure (top) and plot of the double resonance crossing in the tune diagram (bottom). Throughout the double resonance crossing both the sextupole and the octupole were powered with a constant current of $175 \mathrm{~A}$ and $-250 \mathrm{~A}$, respectively.

crossings. The second profile is measured in the middle of a stage with constant tune. Results shown in this paper refer to a stage of constant tune of $20 \mathrm{~ms}$. Measurements repeated with a longer stage $(180 \mathrm{~ms})$ did not show significantly different results. Throughout the double resonance crossing both the sextupole and the octupole used to generate the islands were powered with a constant current of 175 and $-250 \mathrm{~A}$, respectively. It is worth mentioning that the operational beam splitting during the multiturn extraction from the PS will be performed with a variable octupole current, similarly to the one in Fig. 11, the latter providing the best results in terms of losses and vertical emittance growth.

Loss of adiabaticity and reversibility reveals in a final horizontal beam profile with tick tails. By measuring the properties of the latter against the resonance crossing time $T^{*}$, this loss can be quantified. For this purpose, it is hence necessary to define a model for the tails (and the underlying assumptions) to be used for fitting the measured profile. The non-Gaussian tails are interpreted as particles that during the second, reversed, crossing are no longer 
able to follow the fixed points that move towards the center of the horizontal phase space. This may be due to (i) the loss of adiabaticity when the fixed points approach the center, the frequency being inversely proportional to the distance between them; (ii) close to the center the dynamics is completely linear and the fixed points generated by the nonlinear elements fade away. In both cases, the beamlets may survive around the beam core, even after the fixed points disappear. The absence of the latter makes the beamlets to spiral out in the horizontal phase space and hence to form a sort of annulus. This was already observed in the studies of an injection based on beam trapping in stable islands [6]. The final profile would then be the superposition of: (i) A Gaussian central core $\rho_{c}$ containing all particles not captured during the first crossing plus some of the trapped ones that were merged back to the core during the second crossing:

$$
\rho_{c}\left(x, \sigma_{c}, \mu_{c}, A\right)=A e^{-\left[\left(x-\mu_{c}\right)^{2} / 2 \sigma_{c}^{2}\right]},
$$

where $\sigma_{c}$ and $\mu_{c}$ are the RMS core size and centroid, respectively, and $A$ is proportional to the beam intensity in the core. (ii) The projected annulus generated by the survived beamlets. Assuming the beamlets density profile is and remains Gaussian while spiraling out, the projection reads

$$
\begin{aligned}
\rho_{a}\left(x, \sigma_{a}, \mu_{a}, B\right)= & B e^{-\left\{\left[x^{2}+\left(\mu_{a}^{2} / 2\right)\right] / 2 \sigma_{a}^{2}\right\}} 2 \pi \\
& \times\left[I_{0}\left(\frac{\mu_{a}^{2}}{4 \sigma_{s}^{2}}\right) I_{0}\left(\frac{\mu_{a} x}{\sigma_{a}^{2}}\right)\right. \\
& \left.+2 \sum_{k=1}^{\infty}(-1)^{k} I_{k}\left(\frac{\mu_{a}^{2}}{4 \sigma_{a}^{2}}\right) I_{2 k}\left(\frac{\mu_{a} x}{\sigma_{a}^{2}}\right)\right],
\end{aligned}
$$

where $I_{k}$ are the modified Bessel functions of order $k, \sigma_{a}$ is the RMS annulus thickness (or size), $\mu_{a}$ corresponds to the distance at which the particles in the beamlets get detrapped, and $B$ is proportional to the fraction of particles forming the annulus. The analytical proof of Eq. (2) is carried out in the Appendix.

Six parameters can hence be found that best fit the final measured profile with the function $\rho=\rho_{c}+\rho_{a}$. It is worth noting that in the following analysis the summation in Eq. (2) is truncated at $k=6$. The three parameters corresponding to the annulus can be eventually plotted against the crossing time $T^{*}$.

While the initial profile is well fitted by a single Gaussian, for the second one a superposition of a central Gaussian (the beam core) and of four Gaussian functions having the same area (i.e. intensity) is used. The third profile is eventually fitted as described above. Two examples of measurements are reported in Fig. 13. The three beam profiles are shown, for two different crossings of 170 and $20 \mathrm{~ms}$, respectively. While it is natural to have more populated islands when the crossing is slower, it is rather counterintuitive to observe in this case thicker tails, compared to the fast crossing in $20 \mathrm{~ms}$. This is a sign that the
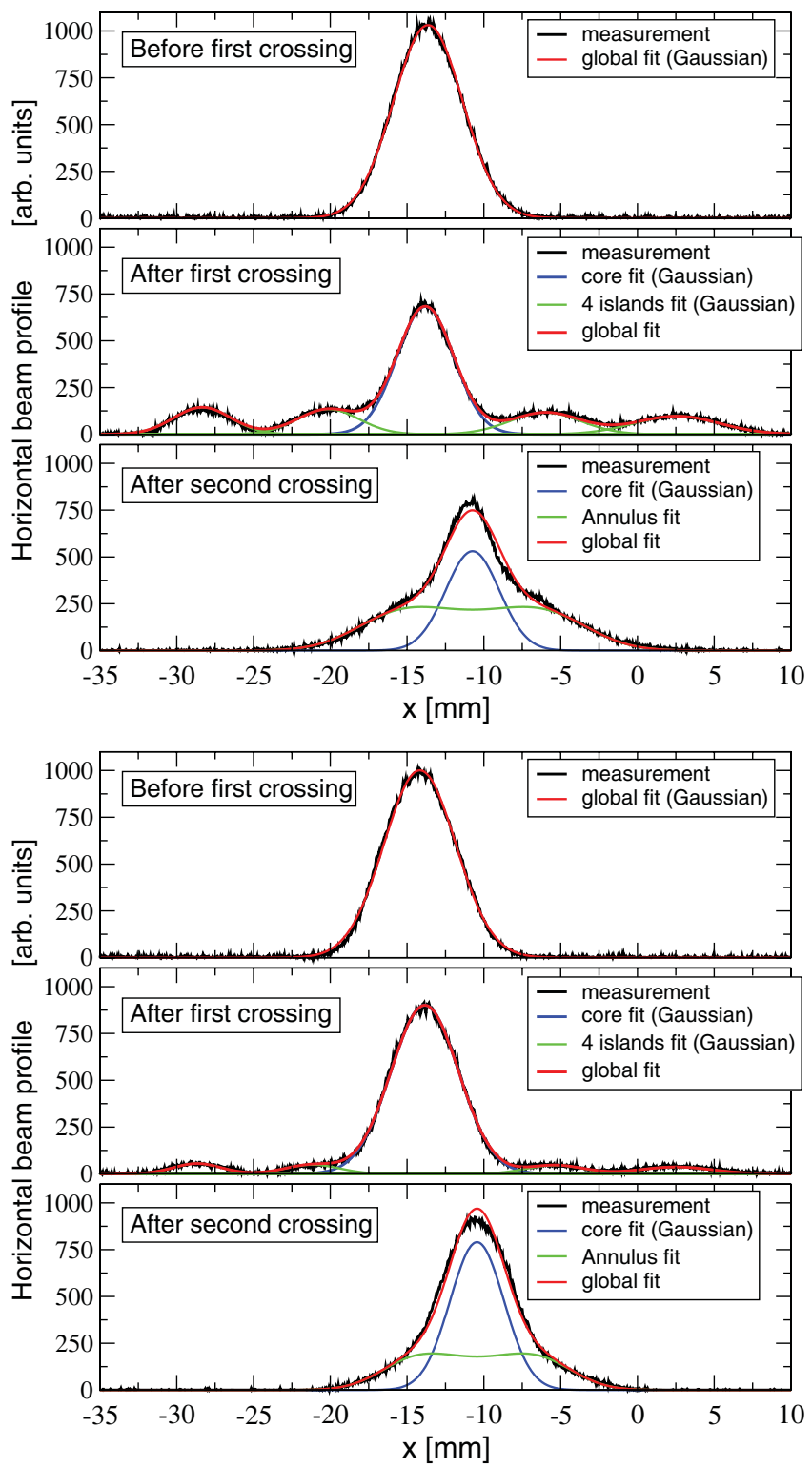

FIG. 13. (Color) Examples of measured horizontal beam profile during the double resonance crossing. For each profile, the various fits are superimposed. The two sets of plots refer to a crossing in $170 \mathrm{~ms}$ (top) and $20 \mathrm{~ms}$ (bottom), respectively.

process is not reversible. Hence, the slower the crossing, the least reversible the process is. The different centroid of the third profile is due to a known offset introduced by the PS wire scanner when more than one measurement is taken during the same cycle.

\section{B. Experimental results}

Data were acquired in 2004 by varying $T^{*}$ from 10 to $170 \mathrm{~ms}$, by steps of $10 \mathrm{~ms}$. A single-bunch beam of $55 \times$ $10^{10}$ protons per bunch, momentum spread $\Delta p / p=1 \times$ $10^{-3}$ at $2 \sigma$, and normalized RMS horizontal emittance $\epsilon_{x}(1 \sigma)=2.1 \mu \mathrm{m}$ was used. From a first analysis it turned out that core RMS size $\sigma_{c}$ was independent from the 

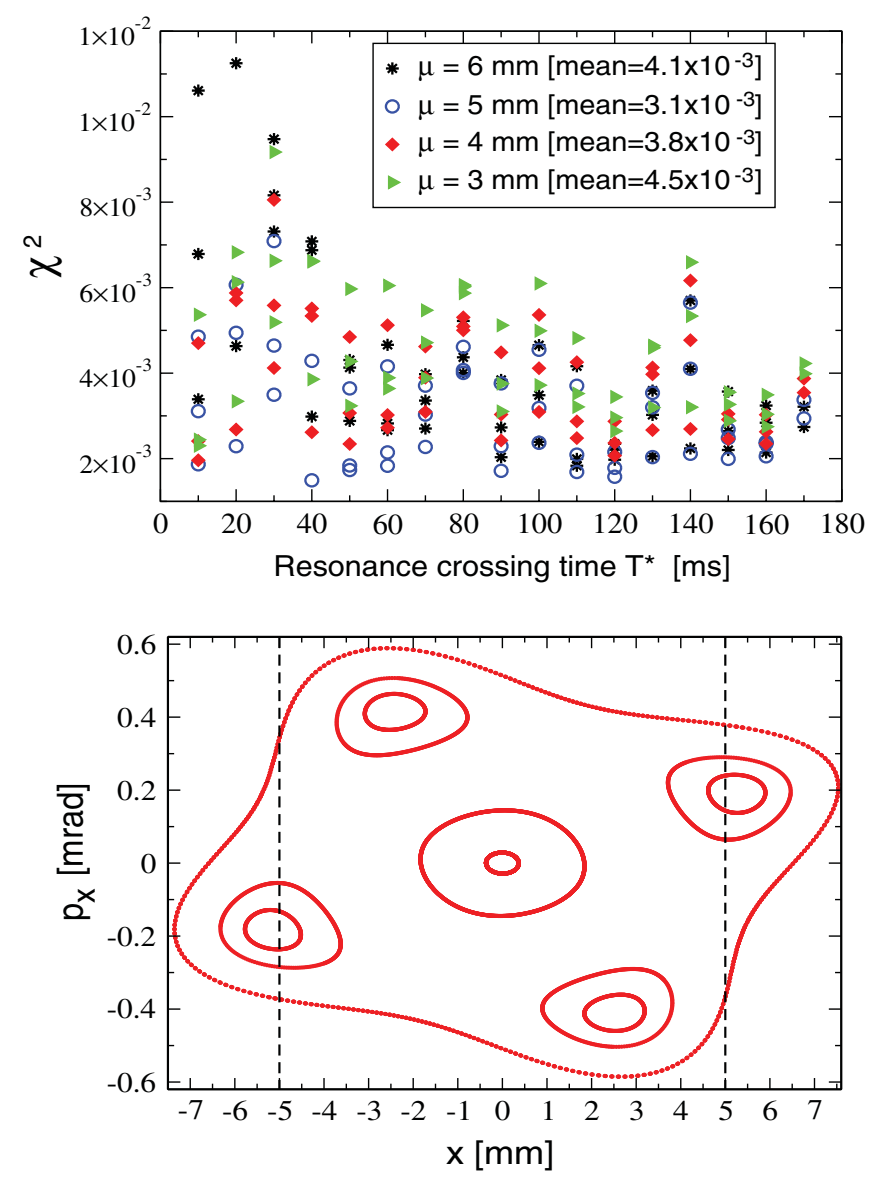

FIG. 14. (Color) Top: $\chi^{2}$ from the four-parameter fit $\rho=\rho_{c}+$ $\rho_{a}$ of the final horizontal beam profile against the resonance crossing time for different values of $\mu_{a}$. For each time $T^{*}$, three data sets were acquired. Bottom: model phase space portrait at $Q_{x}=6.2507$, whose fixed points are located at $5 \mathrm{~mm}$ from the center.

crossing speed $\left(\sigma_{c}=1.75 \mathrm{~mm}\right)$. It was also observed that the fit of $\rho_{a}$ was not unique, different combinations of $\sigma_{a}$ and $\mu_{a}$ providing equivalent global profile. Therefore, it was decided to fix $\mu_{a}$ and to vary the remaining four parameters only. The above considerations (i) and (ii) induce indeed to consider $\mu_{a}$ as a geometrical parameter rather than a dynamical one. After repeating the fit of all data for several values of $\mu_{a}$, the one minimizing the $\chi^{2}$ value was chosen. As shown in the upper plot of Fig. 14, this is achieved for $\mu_{a}=5 \mathrm{~mm}$, that gives an average $\chi^{2}$ of $3.1 \times 10^{-3}$. The PS model of the experimental setup indicates that a horizontal tune $Q_{x}=6.2507$ corresponds to fixed points at $\mu_{a}=5 \mathrm{~mm}$, see bottom plot of Fig. 14 .

In Fig. 15 the fit results are illustrated against the resonance crossing time $T^{*}$. In the upper plot it can be clearly seen how the larger $T^{*}$, the more populated the annulus. For comparison the fraction of particles trapped in the four islands is shown. The particle sharing is inferred from the two fit parameters $A$ and $B$ : the particle fraction in the core reads $A /(A+B)$, while the one in the annulus is equal to

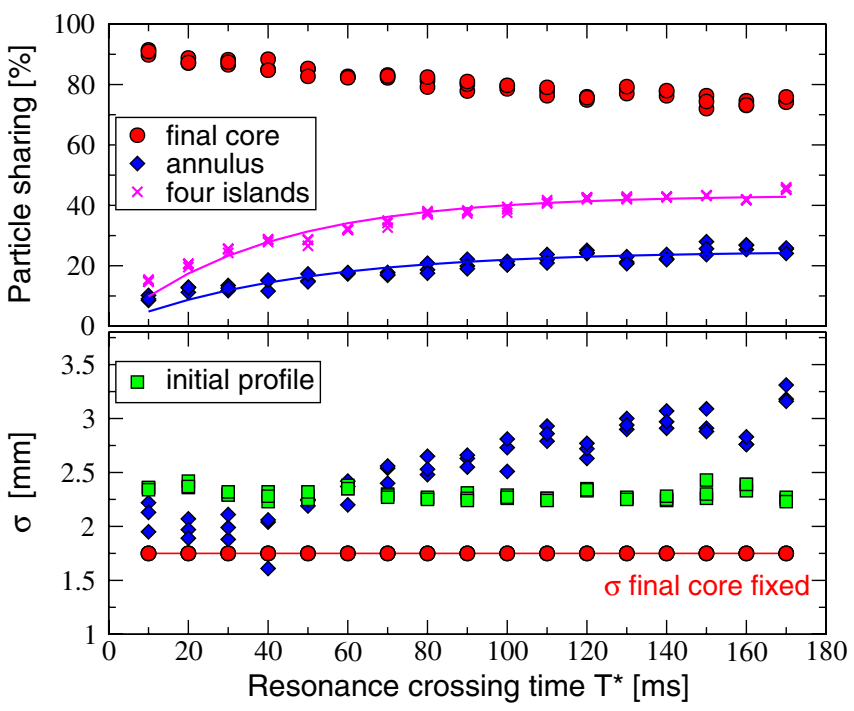

FIG. 15. (Color) Dependence of the particle sharing (top) and of the fit parameter $\sigma$ (bottom) on the resonance crossing time. The fraction of particles is fitted by the curve $y=a\left(1-e^{-b T^{*}}\right)$.

$B /(A+B)$. It is worth mentioning that no particle loss is observed whenever the resonance is double crossed in a time $T^{*}>10 \mathrm{~ms}$. The particle share in the annulus seems to have the same dependence on $T^{*}$ as the one in the four islands, thus indicating that more populated islands are more difficult to merge down into the core. By fitting indeed the two curves with the exponential function $y=$ $a\left(1-e^{-b T^{*}}\right)$, it turns out that both are well fitted by the same time constant $b=24 \pm 2 \mathrm{~s}^{-1}$. For completeness it has to be mentioned that for large $T^{*}$ the islands are not only more populated, but also larger: In Fig. 16 the evolution of the islands' sizes is plotted again $T^{*}$, showing a clear growth. The fact that the RMS sizes are different, while the areas (and the emittances) are the same, is a

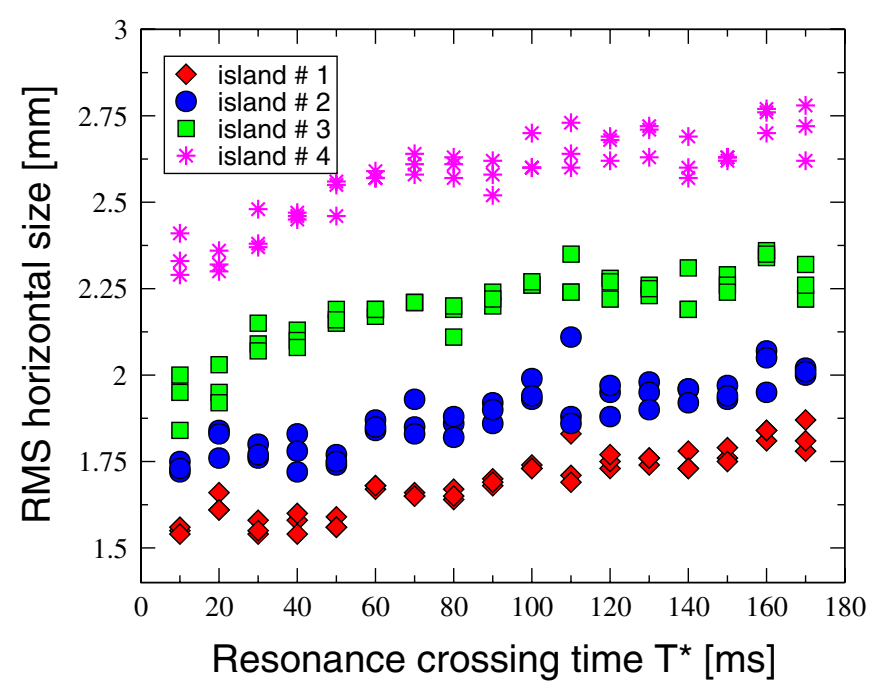

FIG. 16. (Color) Evolution of the islands' RMS sizes against the resonance crossing time $T^{*}$. 
consequence of the nonlinear magnetic fields: The relation $\epsilon_{x}=\sigma_{x}^{2} / \beta_{x}$ does not hold for the islands, the dynamics inside them being nonlinear and each island having a different $\beta_{x}$ around the fixed point. The increase of the islands' size might be the result of both the increased population and the larger beamlets deterioration induced by the prolonged tune modulation driven by chromaticity, the latter already observed in multiparticle simulations of the one-third resonance, see Fig. 8, as well as the constant nonlinear coupling generated by the sextupoles and octupole.

Thicker tails might hence be the consequence of having more particles at large distance from the fixed points that spiral out when the latter collapse onto the center. The lower plot of Fig. 15 shows how the annulus size $\sigma_{a}$ increases for large $T^{*}$. This clearly shows how a longer crossing time improves adiabaticity of the capture process at the expense of reversibility.

Another interesting result is that the size of the beam core after the double crossing, $\sigma_{c}$, is independent from $T^{*}$, while the fraction of particles captured in the islands increases for larger $T^{*}$. By comparing the initial beam size and $\sigma_{c}$ in the lower plot of Fig. 15, it looks like $\sigma_{c}$ defines a sort of region which is transparent to the beam trapping around the moving fixed points, at least with a linear tune ramp used during the experiment.

\section{CONCLUSIONS}

For the first time at the CERN PS beam splitting in stable islands of the horizontal phase space by crossing the unstable one-third and the stable one-fifth resonances was experimentally observed. In the first case an unexpected residual core and losses of about $10 \%$ after the crossing were observed. Multiparticle simulations indicate that a possible explanation for the survival of particles in the central region might be found in the modulation of the horizontal tune induced by the synchrotron motion (the beam used being bunched) via a nonzero chromaticity, that corrupts the adiabatic tune sweep across the resonance. Losses might be related to a not well controlled tune variation after the resonance crossing. It is also important to stress that it was not possible to optimize beam losses essentially due to the limited beam time allocated to these special measurements in the framework of the MTE studies.

A loss-free crossing was performed around the one-fifth resonance, even though the fraction of particles trapped in the five islands is by far lower than in the previous case.

Similar measurements, still with a bunched beam, showed that the beam splitting is an adiabatic process (the longer the resonance crossing time, the larger the number of particles trapped in the islands) but not reversible: Even if the islands merge together towards the center during the inverse resonance crossing, the horizontal beam profile shows thick tails whose population and size in- crease when the crossing speed is reduced. This feature is not explained by the 2D Hénon-like model of the splitting, which is invariant for time reversal. Coupling with the vertical plane and with the synchrotron motion (tune modulation) are considered as possible candidates to explain the loss of reversibility.

\section{ACKNOWLEDGMENTS}

We are grateful to the CERN Operations Group for the support during the measurement campaign.

\section{APPENDIX A: HORIZONTAL BEAM PROFILE OF A ROTATING GAUSSIAN DISTRIBUTION}

In this Appendix the functional form of the horizontal beam profile generated by an off-axis 2D Gaussian distribution after filamentation is derived. The filamentation generates an annulus that, once projected onto the $x$ axis, forms a hollow density profile. The construction of such a distribution is carried out as follows: (i) A normalized uncoupled 2D Gaussian distribution in the phase space reads

$$
\rho\left(\tilde{x}, \tilde{x}^{\prime}, \epsilon_{x}\right)=\frac{1}{2 \pi \epsilon_{x}} e^{-\left[\left(\tilde{x}^{2}+\tilde{x}^{2}\right) / 2 \epsilon_{x}\right]},
$$

where $\tilde{x}, \tilde{x}^{\prime}$ are the canonical variables in the CourantSnyder space [21] and

$$
\epsilon_{x}=\tilde{\sigma}_{x}^{2}=\tilde{\sigma}_{x^{\prime}}^{2}=\sigma_{x}^{2} / \beta_{x},
$$

is the RMS beam emittance (in the linear approximation), and $\beta_{x}$ is the Twiss function. The dispersion term is neglected here. See Fig. 17. (ii) The above distribution in the Courant-Snyder space is then displaced at a certain position $\vec{\mu}$, the latter parametrized by a distance $\tilde{\mu}$ and an angle $\theta$, see Fig. 18. $\tilde{\mu}$ is assumed to be small enough to make Eq. (A2) (i.e. the linear approximation) hold. The

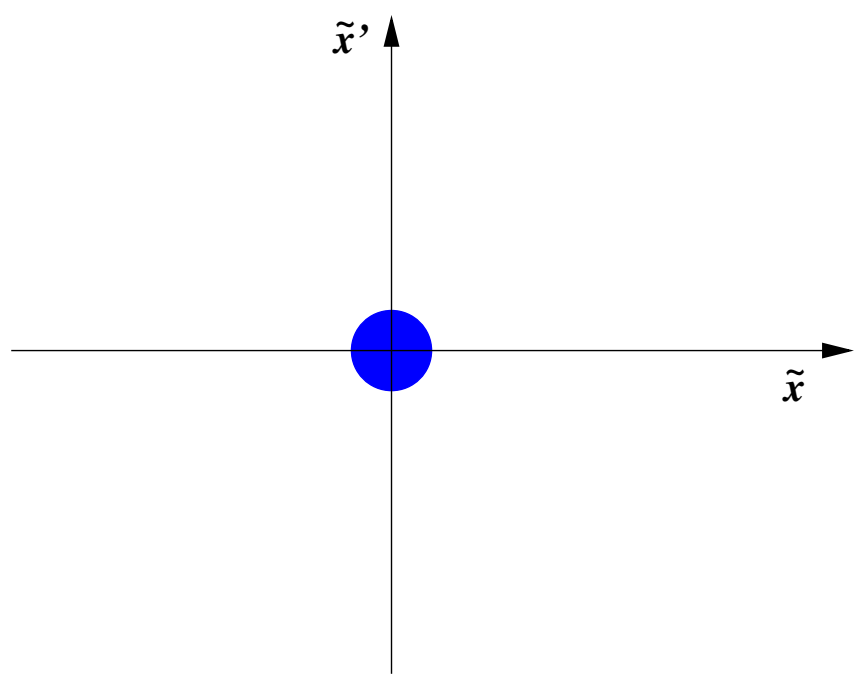

FIG. 17. (Color) A centered Gaussian distribution. 


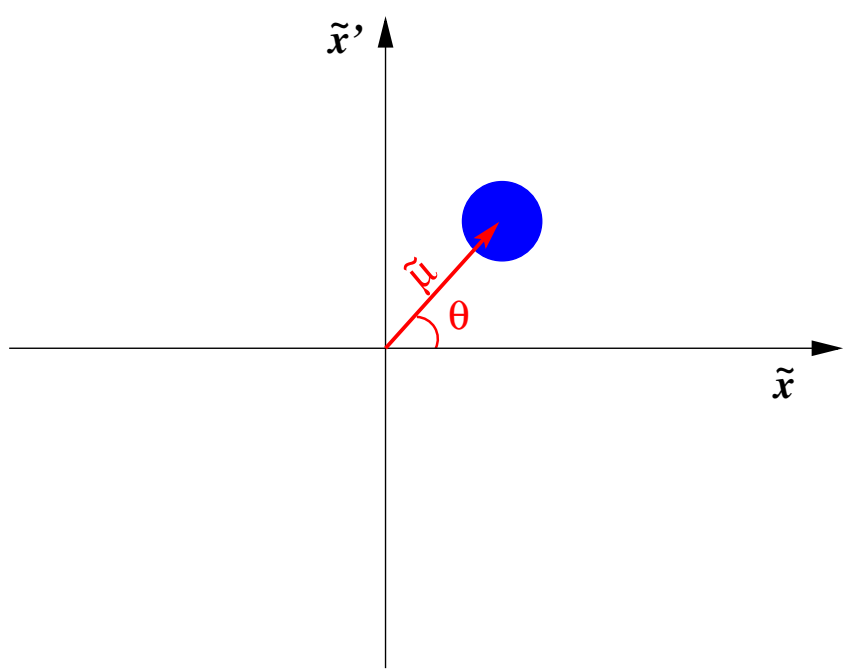

FIG. 18. (Color) A displaced Gaussian distribution.

change of coordinates reads

$$
\tilde{x} \rightarrow \tilde{x}-\tilde{\mu} \cos \theta, \quad \tilde{x}^{\prime} \rightarrow \tilde{x}^{\prime}-\tilde{\mu} \sin \theta .
$$

The distribution becomes then

$$
\rho\left(\tilde{x}, \tilde{x}^{\prime}, \boldsymbol{\epsilon}_{x}, \tilde{\mu}, \theta\right)=\frac{1}{2 \pi \epsilon_{x}} e^{\left.-\left\{(\tilde{x}-\tilde{\mu} \cos \theta)^{2}+\left(\tilde{x}^{\prime}-\tilde{\mu} \sin \theta\right)^{2}\right] / 2 \epsilon_{x}\right\}} .
$$

(iii) The annulus is created by integrating the above function over $\theta$ from 0 to $2 \pi$, see Fig. 19, namely

$$
\rho\left(\tilde{x}, \tilde{x}^{\prime}, \epsilon_{x}, \tilde{\mu}\right)=\int_{0}^{2 \pi} \rho\left(\tilde{x}, \tilde{x}^{\prime}, \epsilon_{x}, \tilde{\mu}, \theta\right) d \theta .
$$

(iv) The corresponding horizontal beam profile is obtained by further integrating over $\tilde{x}^{\prime}$, see Fig. 20:

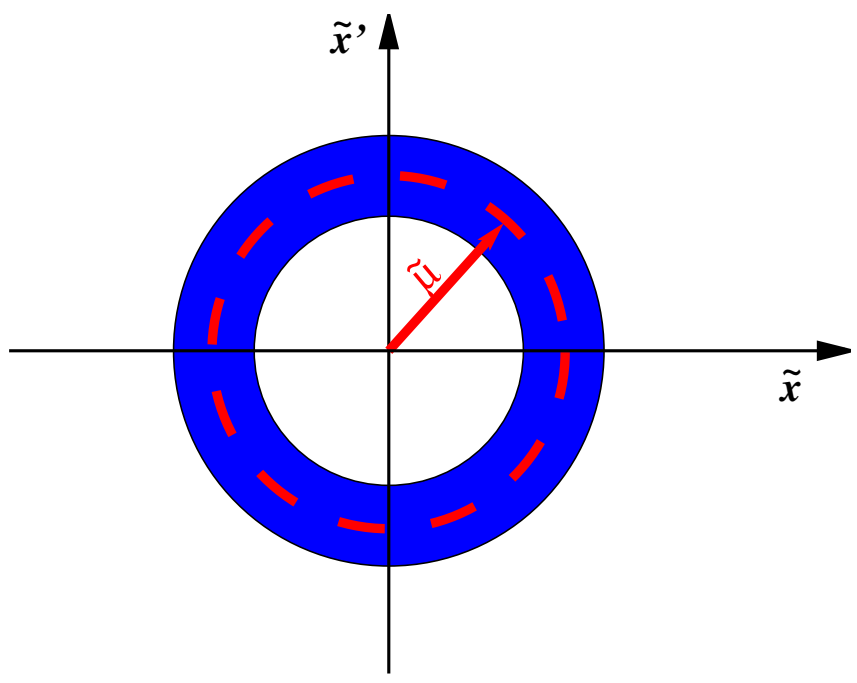

FIG. 19. (Color) A Gaussian annulus distribution.

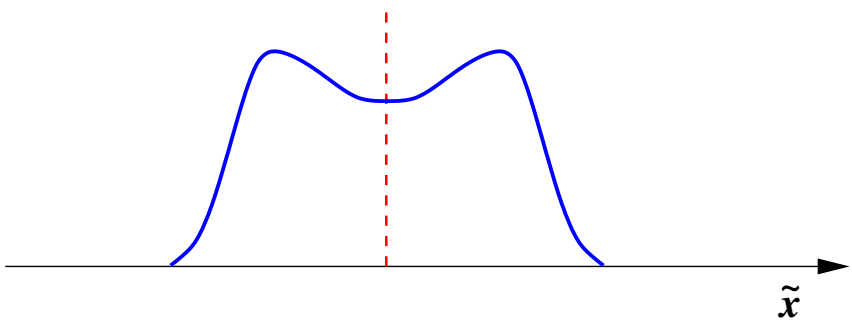

FIG. 20. (Color) Beam profile (annulus projection on $x$ ).

$$
\rho\left(\tilde{x}, \boldsymbol{\epsilon}_{x}, \tilde{\mu}\right)=\int_{-\infty}^{\infty} \rho\left(\tilde{x}, \tilde{x}^{\prime}, \boldsymbol{\epsilon}_{x}, \tilde{\mu}\right) d \tilde{x}^{\prime} .
$$

(v) The inverse Courant-Snyder transformation is eventually performed,

$$
x=\tilde{x} \sqrt{\beta_{x}}, \quad \sigma_{x}=\sqrt{\epsilon_{x} \beta_{x}},
$$

to write the beam profile as function of observables, $\rho\left(x, \sigma_{x}, \mu\right)$.

Steps (i)-(iv) can be grouped in the following integral:

$$
\begin{aligned}
\rho\left(\tilde{x}, \boldsymbol{\epsilon}_{x}, \tilde{\mu}\right)= & \int_{0}^{2 \pi} \int_{\infty}^{\infty} \rho\left(\tilde{x}, \tilde{x}^{\prime}, \epsilon_{x}, \tilde{\mu}\right) d \tilde{x}^{\prime} d \theta \\
= & \int_{0}^{2 \pi} \frac{1}{\sqrt{2 \pi \epsilon_{x}}} e^{-\left[(\tilde{x}-\tilde{\mu} \cos \theta)^{2} / 2 \epsilon_{x}\right]} \\
& \times\left(\int_{-\infty}^{\infty} \frac{1}{\sqrt{2 \pi \epsilon_{x}}} e^{-\left[\left(\tilde{x}^{\prime}-\tilde{\mu} \sin \theta\right)^{2} / 2 \epsilon_{x}\right]} d \tilde{x}^{\prime}\right) d \theta .
\end{aligned}
$$

The integral over $\tilde{x}^{\prime}$ in the brackets is equal to one, since the initial distribution is normalized and the $\tilde{\mu} \sin \theta$ term represents a shift that does not alter the normalization. The argument of the first exponential term reads

$$
\begin{aligned}
(\tilde{x}-\tilde{\mu} \cos \theta)^{2} & =\tilde{x}^{2}+\tilde{\mu}^{2} \cos \theta^{2}-2 \tilde{x} \tilde{\mu} \cos \theta \\
& =\tilde{x}^{2}+\frac{\tilde{\mu}^{2}}{2}+\frac{\tilde{\mu}^{2}}{2} \cos 2 \theta-2 \tilde{x} \tilde{\mu} \cos \theta,
\end{aligned}
$$

where the trigonometric relation $\cos ^{2} \theta=1 / 2(1+\cos 2 \theta)$ has been used. Equation (A8) can be then rewritten as

$$
\begin{aligned}
\rho\left(\tilde{x}, \epsilon_{x}, \tilde{\mu}\right)= & \frac{1}{\sqrt{2 \pi \epsilon_{x}}} e^{-\left\{\left[\tilde{x}^{2}+\left(\tilde{\mu}^{2} / 2\right)\right] / 2 \epsilon_{x}\right\}} \\
& \times \int_{0}^{2 \pi} e^{-\left\{\left[\left(\tilde{\mu}^{2} / 2\right) \cos 2 \theta-2 \tilde{x} \tilde{\mu} \cos \theta\right] / 2 \epsilon_{x}\right\}} d \theta .
\end{aligned}
$$

It is straightforward to prove that applying the coordinate change of Eq. (A7) leads to

$$
\begin{aligned}
\rho\left(x, \sigma_{x}, \mu\right)= & B e^{-\left\{\left[x^{2}+\left(\mu^{2} / 2\right)\right] / 2 \sigma_{x}^{2}\right\}} \\
& \times \int_{0}^{2 \pi} e^{-\left\{\left[\left(\mu^{2} / 2\right) \cos 2 \theta-2 x \mu \cos \theta\right] / 2 \sigma_{x}^{2}\right\}} d \theta .
\end{aligned}
$$

The normalization factor $B=\int \rho d x$ is introduced, since 


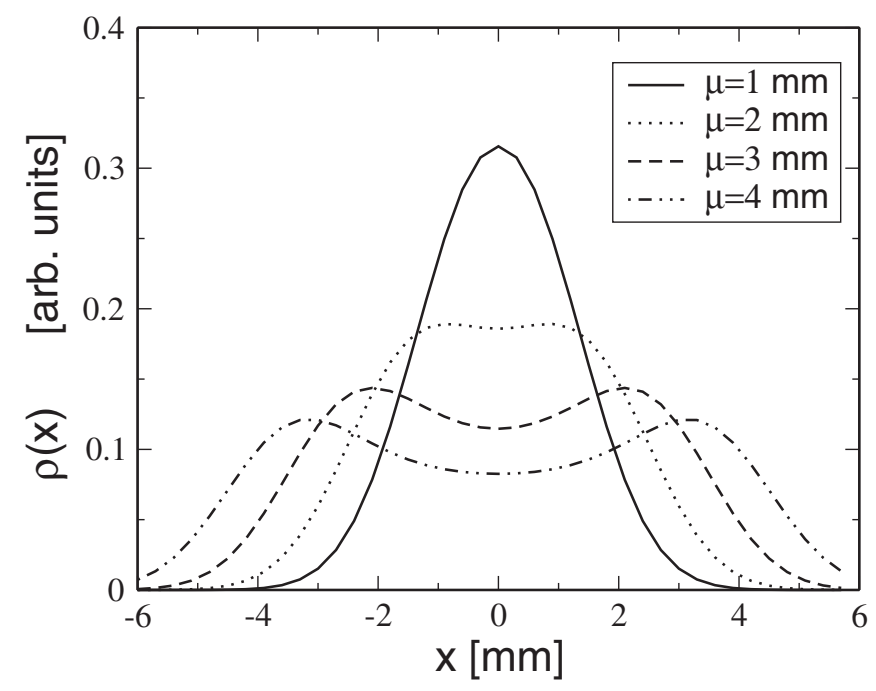

FIG. 21. Example of the beam profile of Eq. (A11) for $\sigma_{x}=$ $1 \mathrm{~mm}$ and several values of $\mu$.

the distribution of Eq. (A10) is no longer normalized. While $x$ can be obtained directly from the measured profile, $B, \sigma_{x}$, and $\mu$ shall be inferred from a fit. In Fig. 21 an example of such a profile is plotted for $\sigma_{x}=1 \mathrm{~mm}$ and several values of $\mu$ : Shoulder appears when $\mu>\sigma_{x}$.

A more handy expression of Eq. (A11) can be obtained by expanding the above integral as a series of modified Bessel functions $I_{n}$. For this, the following relations are used:

$$
e^{z \cos n \theta}=I_{0}(z)+2 \sum_{k=1}^{\infty} I_{k}(z) \cos [k(n \theta)], \quad \text { for } z>0
$$

$$
I_{k}(-z)=(-1)^{k} I_{k}(z)
$$

While the sign of the first term in the argument of the exponential in the integral of Eq. (A11) is fixed, $\mu^{2} / 2<0$, the sign of the second term $(2 x \mu)$ depends on the sign of $x$. By construction, the beam profile is however symmetric with respect to $x=0$. We can therefore limit the expansion to the positive axis $(x>0)$. By doing so, the signs of the two terms are always opposite. By making use of Eqs. (A12) and (A13), the integral of Eq. (A11) can be cast in the following form:

$$
\begin{gathered}
\int_{0}^{2 \pi} e^{-\left\{\left[\left(\mu^{2} / 2\right) \cos 2 \theta-2 x \mu \cos \theta\right] / 2 \sigma_{x}^{2}\right\}} d \theta \\
=C_{1}+C_{2}+C_{3}+C_{4}, \\
C_{1}=2 \pi I_{0}\left(T_{1}\right) I_{0}\left(T_{2}\right), \\
C_{2}=2 I_{0}\left(T_{1}\right) \sum_{j=1}^{\infty} I_{j}\left(T_{2}\right) \int_{0}^{2 \pi} \cos j \theta d \theta=0,
\end{gathered}
$$

$$
\begin{gathered}
C_{3}=2 I_{0}\left(T_{2}\right) \sum_{k=1}^{\infty}(-1)^{k} I_{k}\left(T_{1}\right) \int_{0}^{2 \pi} \cos 2 k \theta d \theta=0, \\
C_{4}=4 \sum_{j, k=1}^{\infty}(-1)^{k} I_{k}\left(T_{1}\right) I_{j}\left(T_{2}\right) \int_{0}^{2 \pi} \cos 2 k \theta \cos j \theta d \theta \\
=4 \pi \sum_{k=1}^{\infty}(-1)^{k} I_{k}\left(T_{1}\right) I_{2 k}\left(T_{2}\right), \\
T_{1}=\frac{\mu^{2}}{4 \sigma_{x}^{2}}, \quad T_{2}=\frac{\mu x}{\sigma_{x}^{2}} .
\end{gathered}
$$

Hence the integral of Eq, (A11) can be eventually written as

$$
\begin{aligned}
& \int_{0}^{2 \pi} e^{-\left\{\left[\left(\mu^{2} / 2\right) \cos 2 \theta-2 x \mu \cos \theta\right] / 2 \sigma_{x}^{2}\right\}} d \theta \\
& \quad=2 \pi\left[I_{0}\left(T_{1}\right) I_{0}\left(T_{2}\right)+2 \sum_{k=1}^{\infty}(-1)^{k} I_{k}\left(T_{1}\right) I_{2 k}\left(T_{2}\right)\right] .
\end{aligned}
$$

After replacing $T_{1}$ and $T_{2}$ from Eq. (A19), the horizontal beam profile reads

$$
\begin{aligned}
\rho(x, \sigma, \mu, B)= & B e^{-\left\{\left[x^{2}+\left(\mu^{2} / 2\right)\right] / 2 \sigma^{2}\right\}} 2 \pi \\
& \times\left[I_{0}\left(\frac{\mu^{2}}{4 \sigma_{s}^{2}}\right) I_{0}\left(\frac{\mu x}{\sigma^{2}}\right)\right. \\
& \left.+2 \sum_{k=1}^{\infty}(-1)^{k} I_{k}\left(\frac{\mu^{2}}{4 \sigma^{2}}\right) I_{2 k}\left(\frac{\mu x}{\sigma^{2}}\right)\right] .
\end{aligned}
$$

In Fig. 22 the difference between the profile of Eq. (A11) and the expansion of Eq. (A21) is plotted for $\sigma_{x}=1 \mathrm{~mm}$ and several truncation orders $K$ of the series in the latter equation.

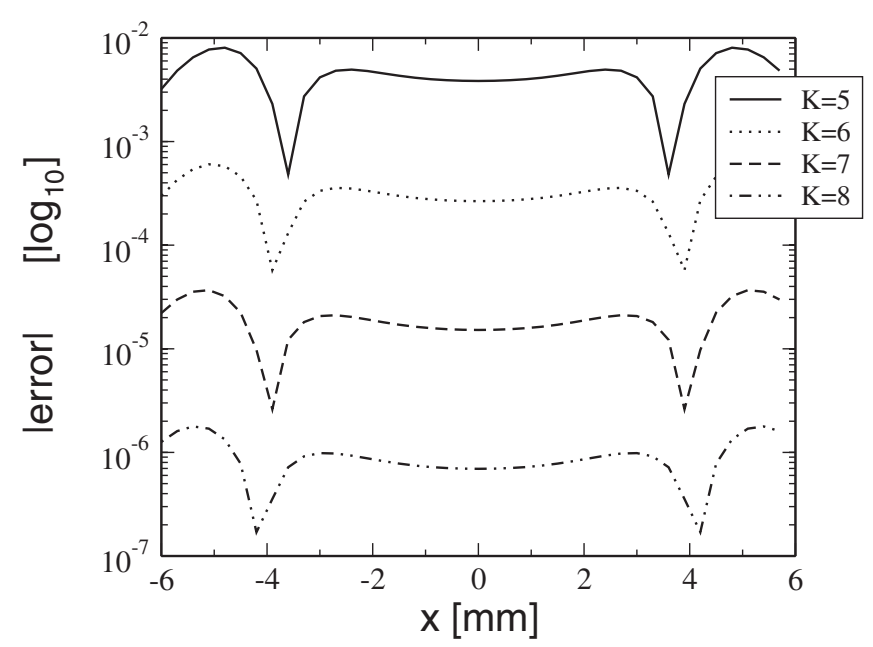

FIG. 22. Absolute value of the difference between Eq. (A11) and Eq. (A21) for $\sigma_{x}=1 \mathrm{~mm}, \mu=1 \mathrm{~mm}$, and several truncation orders $K$. 
[1] R. Cappi and M. Giovannozzi, Phys. Rev. Lett. 88, 104801 (2002).

[2] R. Cappi and M. Giovannozzi, Phys. Rev. ST Accel. Beams 7, 024001 (2004).

[3] M.-J. Barnes, O.-E. Berrig, A. Beuret, J. Borburgh, P. Bourquin, R. Brown, J.-P. Burnet, F. Caspers, J.-M. Cravero, T. Dobers, T. Fowler, S. Gilardoni, M. Hourican, W. Kalbreier, T. Kroyer, F. di Maio, M. Martini, V. Mertens, E. Métral, K.-D. Metzmacher, C. Rossi, J.-P. Royer, L. Sermeus, R. Steerenberg, G. Villiger, and T. Zickler, CERN Report, 2006-011, edited by M. Giovannozzi, 2006.

[4] C. Bovet, D. Fiander, L. Henni, A. Krusche, and G. Plasse, Proceedings of the 1973 Particle Accelerator Conference, edited by D. W. Dupen (IEEE Computer Society Press, New York, 1973), p. 438.

[5] R. Cappi and M. Giovannozzi, CERN Report No. PS2002-083 (AE), 2002.

[6] M. Giovannozzi and J. Morel, Phys. Rev. ST Accel. Beams 10, 034001 (2007).

[7] S. Gilardoni, M. Giovannozzi, M. Martini, E. Métral, P. Scaramuzzi, R. Steerenberg, and A.-S. Müller, Phys. Rev. ST Accel. Beams 9, 104001 (2006).

[8] S. Gilardoni, M. Giovannozzi, M. Martini, E. Métral, P. Scaramuzzi, R. Steerenberg, and A.-S. Müller, Nucl. Instrum. Methods Phys. Res., Sect. A 561, 249 (2006).

[9] G. Acquistapace, J.-L. Baldy, A.-E. Ball, P. Bonnal, M. Buhler-Broglin, F. Carminati, E. Cennini, A. Ereditato, V.P. Falaleev, P. Faugeras, A. Ferrari, G. Fortuna, L. Foá, R. Genand, A.-L. Grant, L. Henny, A. Hilaire, K. Hübner, J. Inigo-Golfin, K.-H. Kissler, L.-A. López-Hernandez, J.-M. Maugain, M. Mayoud, P. Migliozzi, D. Missiaen, V. Palladino, I.-M. Papadopoulos, F. Pietropaolo, S. Péraire, S. Rangod, J.-P. Revol, J. Roche, P.-R. Sala, C. Sanelli, G. R. Stevenson, B. Tomat, E. Tsesmelis, R. Valbuena, H.-H. Vincke, E. Weisse, and M. Wilhelmsson, CERN 98-02, edited by K. Elsener, 1998.
[10] M. Hénon, Q. Appl. Math. 27, 291 (1969).

[11] V. Kiselev, E. Levichev, O. Meshkov, S. Nikitin, and P. Piminov, in Proceedings of the 11th European Particle Accelerator Conference, Genoa, 2008 (EPS-AG, Genoa, Italy, 2008), p. 965.

[12] T. Risselada, CERN PS (PA) Note 92-04, 1992.

[13] D. Cornuet and Z. Sharifullin, CERN AT (MA) Note 9223, 1992.

[14] J.-P. Burnet, M. Giovannozzi, E. Métral, O. Michels, R. Steerenberg, and B. Vandorpe, in Proceedings of the 10th European Particle Accelerator Conference, Edinburgh, Scotland, 2006 (EPS-AG, Edinburgh, Scotland, 2006), p. 264.

[15] R. Cappi, M. Giovannozzi, M. Martini, E. Métral, G. Métral, R. Steerenberg, and A.-S. Müller, in Proceedings of the 20th Particle Accelerator Conference, Portland, OR, 2003 (IEEE, New York, 2003), p. 388.

[16] H. Grote and F. Schmidt, Proceedings of the 20th Particle Accelerator Conference, Portland, OR, 2003, Ref. [15], p. 3497.

[17] E. Forest, F. Schmidt, and E. McIntosh, in Proceedings of the 21st Particle Accelerator Conference, Knoxville, 2005 (IEEE, Piscataway, NJ, 2005); F. Schmidt, in Proceedings of the 21st Particle Accelerator Conference, Knoxville, 2005, p. 1272.

[18] V. Agoritsas, E. Falk, F. Hoekemeijer, J. Olsfors, and C. Steinbach, CERN-PS-95-06-BD-OP, 1995.

[19] S. Gilardoni and J. Barranco, Proceedings of the 11th European Particle Accelerator Conference, Genoa, 2008, Ref. [11], p. 3083.

[20] V. Agoritsas, F. Beck, G. P. Benincasa, and J. P. Bovigny, Nucl. Instrum. Methods Phys. Res., Sect. A 247, 44 (1986).

[21] E. Courant and H. Snyder, Ann. Phys. (N.Y.) 3, 1 (1958).

[22] M. Juchno (private communication). 